\title{
Four-neutrino oscillation solutions of the solar neutrino problem
}

\author{
C. Giunti \\ INFN, Sez. di Torino, and Dip. di Fisica Teorica, Univ. di Torino, I-10125 Torino, Italy \\ M. C. Gonzalez-Garcia and C. Peña-Garay \\ Instituto de Física Corpuscular (IFIC) CSIC-Universidad de Valencia, Edificio Institutos de Paterna, \\ Apartado 2085, 46071 Valencia, Spain \\ (Received 12 January 2000; published 6 June 2000)
}

\begin{abstract}
We present an analysis of the neutrino oscillation solutions of the solar neutrino problem in the framework of four-neutrino mixing where a sterile neutrino is added to the three standard ones. We perform a fit to the full data set corresponding to the 825-day Super-Kamiokande data sample as well as to chlorine, GALLEX, and SAGE and Kamiokande experiments. In our analysis we use all measured total event rates as well as all Super-Kamiokande data on the zenith angle dependence and the recoil electron energy spectrum. We consider both transitions via the Mikheyev-Smirnov-Wolfenstein (MSW) mechanism as well as oscillations in vacuum (just-so) and find the allowed solutions for different values of the additional mixing angles. This framework permits transitions into active or sterile neutrinos controlled by the additional parameter $\cos ^{2}\left(\vartheta_{23}\right) \cos ^{2}\left(\vartheta_{24}\right)$ and contains as limiting cases the pure $\nu_{e}$-active and $\nu_{e}$-sterile neutrino oscillations. We discuss the maximum allowed values of this additional mixing parameter for the different solutions. As a particularity, we also show that for MSW transitions there are solutions at $99 \%$ C.L. at $\vartheta_{12}$ mixing angles greater than $\pi / 4$ and that the best-fit point for the zenith angle distribution is in the second octant.
\end{abstract}

PACS number(s): 26.65. $+\mathrm{t}, 13.15 .+\mathrm{g}, 14.60 . \mathrm{Pq}, 96.60 . \mathrm{Jw}$

\section{INTRODUCTION}

Solar neutrinos were first detected three decades ago in the Homestake experiment [1] and from the very beginning the puzzling issue of the deficit in the observed rate as compared to the theoretical expectation based on the standard solar model [2] was pointed out, with the implicit assumption that neutrinos created in the solar interior reach the Earth unchanged; i.e., they are massless and have only standard properties and interactions. This discrepancy led to a change in the original goal of using solar neutrinos to probe the properties of the solar interior towards the study of the properties of the neutrino itself and it triggered an intense activity both theoretical as well as experimental, with new measurements being proposed in order to address the origin of the deficit.

On the theoretical side, enormous progress has been made in the improvement of solar modeling and calculation of nuclear cross sections. For example, helioseismological observations have now established that diffusion is occurring and by now most solar models incorporate the effects of helium and heavy element diffusion $[3,4]$. From the experimental point of view the situation is now much richer. Four additional experiments to the original chlorine experiment at Homestake [5] have also detected solar neutrinos: the radiochemical gallium experiments on $p p$ neutrinos, GALLEX [6] and SAGE [7], and the water Cherenkov detectors Kamiokande [8] and Super-Kamiokande $[9,10]$. The latter have been able, not only to confirm the original detection of solar neutrinos at lower rates than predicted by standard solar models, but also to demonstrate directly that the neutrinos come from the sun by showing that recoil electrons are scattered in the direction along the sun-earth axis. Moreover, they have also provided us with good information on the time dependence of the event rates during the day and night, as well as a measurement of the recoil electron energy spectrum. After 825 days of operation, Super-Kamiokande has also presented preliminary results on the seasonal variation of the neutrino event rates, an issue which will become important in discriminating the Mikheyev-SmirnovWolfenstein (MSW) scenario from the possibility of neutrino oscillations in vacuum $[11,12]$. At the present stage, the quality of the experiments themselves and the robustness of the theory give us confidence that in order to describe the data one must depart from the standard model (SM) of particle physics interactions by endowing neutrinos with new properties. In theories beyond the SM, neutrinos may naturally have new properties, the most generic of which is the existence of mass. It is undeniable that the most popular explanation of the solar neutrino anomaly is in terms of neutrino masses and mixing leading to neutrino oscillations either in vacuum [13] or via the matter-enhanced MSW mechanism [14].

On the other hand, together with the results from the solar neutrino experiments we have more evidence pointing towards the existence of neutrino masses and mixing: the atmospheric neutrino data and the Liquid Scintillation Neutrino Detector (LSND) results. The first one can be summarized in the existence of a long-standing anomaly between the predicted and observed $\nu_{\mu} / \nu_{e}$ ratio of the atmospheric neutrino fluxes [15]. In this respect, the confirmation by the Super-Kamiokande collaboration $[10,16]$ of the atmospheric neutrino zenith-angle-dependent deficit, which strongly indicates the existence of $\nu_{\mu}$ conversion, has been of crucial relevance. In addition to the solar and atmospheric neutrino results from underground experiments, there is also an indication that there are neutrino oscillations in the $\bar{\nu}_{\mu}$

$\rightarrow \bar{\nu}_{e}$ channel by the LSND experiment [17]. All these experimental results can be accommodated in a single neutrino 
oscillation framework only if there are at least three different scales of neutrino mass-squared differences. The simplest case of three independent mass-squared differences requires the existence of a light sterile neutrino, i.e., one whose interaction with standard model particles is much weaker than the $\mathrm{SM}$ weak interaction, so it does not affect the invisible $\mathrm{Z}$ decay width, precisely measured at the CERN $e^{+} e^{-}$collider LEP [18-22].

In this paper we present an analysis of the neutrino oscillation solutions of the solar neutrino problem in the framework of four-neutrino mixing where a sterile neutrino is added to the three standard ones. We perform a fit of the full data set corresponding to the 825-day Super-Kamiokande data sample as well as the data of the chlorine, GALLEX, and SAGE experiments. In our analysis we use all measured total event rates and all Super-Kamiokande data on the zenith angle dependence and the recoil electron energy spectrum. We consider both transitions via the MikheyevSmirnov-Wolfenstein (MSW) mechanism as well as oscillations in vacuum (just-so) and find the allowed solutions for different values of the additional mixing angles. Our analysis contains as limiting cases the pure $\nu_{e}$-active and $\nu_{e}$-sterile neutrino oscillations. We discuss the maximum allowed values of the additional mixing angles for which the different solutions are allowed.

The outline of the paper is as follows. In Sec. II we summarize the main expressions for the neutrino oscillation formulas that we use in the analysis of solar neutrino data which take into account matter effects in the case of the MSW solution of the solar neutrino problem. We also present some improvement concerning the calculation of the regeneration of solar $\nu_{e}$ 's in the Earth in the Appendix. Section III contains the summary of our calculations for the predictions of the different observables. Our quantitative results for the analysis of the four-neutrino oscillation parameters are given in Sec. IV. Finally, in Sec. V, we summarize and discuss briefly our conclusions.

\section{FOUR-NEUTRINO OSCILLATIONS}

In this paper we consider the two four-neutrino schemes that can accommodate the results of all neutrino oscillation experiments $[20,21]$ :

(A)
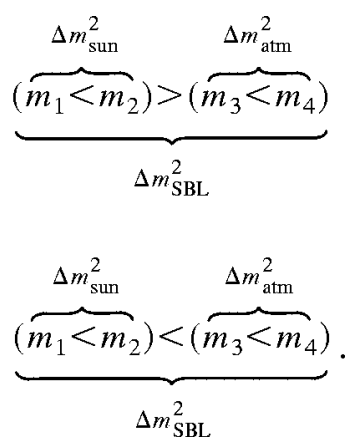

In both these mass spectra there are two pairs of close masses separated by a gap of about $1 \mathrm{eV}$ which gives the mass-squared difference $\Delta m_{\mathrm{SBL}}^{2}=\Delta m_{41}^{2}$ responsible for the short-baseline (SBL) oscillations observed in the LSND experiment (we use the common notation $\Delta m_{k j}^{2} \equiv m_{k}^{2}-m_{j}^{2}$ ). We have ordered the masses in such a way that in both schemes $\Delta m_{\text {sun }}^{2}=\Delta m_{21}^{2}$ produces solar neutrino oscillations and $\Delta m_{\mathrm{atm}}^{2}=\Delta m_{43}^{2}$ is responsible for atmospheric neutrino oscillations. With this convention, the data of solar neutrino experiments can be analyzed using the neutrino oscillation formalism presented in Ref. [22], that takes into account matter effects. In this section we present the neutrino oscillation formulas that we use in the analysis of solar neutrino data. The transition probabilities that take into account matter effects in the case of the MSW solution of the solar neutrino problem have been derived in Ref. [22]. Here we present some improvement concerning the calculation of the regeneration of solar $\nu_{e}$ 's in the Earth (see the Appendix).

In four-neutrino schemes the flavor neutrino fields $\nu_{\alpha L}$ $(\alpha=e, s, \mu, \tau)$ are related to the fields $\nu_{k L}$ of neutrinos with masses $m_{k}$ by the relation

$$
\nu_{\alpha L}=\sum_{k=1}^{4} U_{\alpha k} \nu_{k L} \quad(\alpha=e, s, \mu, \tau),
$$

where $U$ is a $4 \times 4$ unitary mixing matrix, for which we choose the parametrization

$$
U=U_{34} U_{24} U_{23} U_{14} U_{13} U_{12},
$$

where

$$
\begin{aligned}
\left(U_{i j}\right)_{a b}= & \delta_{a b}+\left(\cos \vartheta_{i j}-1\right)\left(\delta_{i a} \delta_{i b}+\delta_{j a} \delta_{j b}\right) \\
& +\sin \vartheta_{i j}\left(\delta_{i a} \delta_{j b}-\delta_{j a} \delta_{i b}\right)
\end{aligned}
$$

represents a rotation in the $i-j 2 \times 2$ sector by an angle $\vartheta_{i j}$. In the parametrization (2.3) we have neglected, for simplicity, the possible presence of $C P$-violating phases.

Since the negative results of the Bugey $\bar{\nu}_{e}$ disappearance experiment [33] imply that $\left|U_{e 3}\right|^{2}+\left|U_{e 4}\right|^{2} \lesssim 3 \times 10^{-2}$ for $\Delta m_{\mathrm{SBL}}^{2}$ in the LSND-allowed region $0.2 \mathrm{eV}^{2} \leqslant \Delta m_{\mathrm{SBL}}^{2}$ $\$ 2 \mathrm{eV}^{2}$, in the study of solar neutrino oscillations the matrices $U_{13}$ and $U_{14}$ can be approximated with the unit matrix (i.e., $\vartheta_{13}=\vartheta_{14}=0$ ) and we obtain

$$
U=U_{34} U_{24} U_{23} U_{12}
$$

Explicitly, we have 


$$
U=\left(\begin{array}{cccc}
c_{12} & s_{12} & 0 & 0 \\
-s_{12} c_{23} c_{24} & c_{12} c_{23} c_{24} & s_{23} c_{24} & s_{24} \\
s_{12}\left(c_{23} s_{24} s_{34}+s_{23} c_{34}\right) & -c_{12}\left(s_{23} c_{34}+c_{23} s_{24} s_{34}\right) & c_{23} c_{34}-s_{23} s_{24} s_{34} & c_{24} s_{34} \\
s_{12}\left(c_{23} s_{24} c_{34}-s_{23} s_{34}\right) & c_{12}\left(s_{23} s_{34}-c_{23} s_{24} c_{34}\right) & -\left(c_{23} s_{34}+s_{23} s_{24} c_{34}\right) & c_{24} c_{34}
\end{array}\right),
$$

where $\boldsymbol{\vartheta}_{12}, \vartheta_{23}, \vartheta_{24}, \vartheta_{34}$ are four mixing angles and $c_{i j}$ $\equiv \cos \vartheta_{i j}$ and $s_{i j} \equiv \sin \vartheta_{i j}$.

Since solar neutrino oscillations are generated by the mass-square difference between $\nu_{2}$ and $\nu_{1}$, it is clear from Eq. (2.6) that the survival of solar $\nu_{e}$ 's mainly depends on the mixing angle $\vartheta_{12}$, whereas the mixing angles $\vartheta_{23}$ and $\vartheta_{24}$ determine the relative amount of transitions into sterile $\nu_{S}$ or active $\nu_{\mu}$ and $\nu_{\tau}$. Let us remind the reader that $\nu_{\mu}$ and $\nu_{\tau}$ cannot be distinguished in solar neutrino experiments, because their matter potential and their interaction in the detectors are equal, due only to neutral-current weak interactions. The active and/or sterile ratio and solar neutrino oscillations in general do not depend on the mixing angle $\vartheta_{34}$, which contributes only to the different mixings of $\nu_{\mu}$ and $\nu_{\tau}$, and depends on the mixing angles $\vartheta_{23} \vartheta_{24}$ only through the combination $\cos \vartheta_{23} \cos \vartheta_{24}$. Indeed, from Eq. (2.6) one can see that the mixing of $\nu_{s}$ with $\nu_{1}$ and $\nu_{2}$ depends only on $\vartheta_{12}$ and the product $\cos \vartheta_{23} \cos \vartheta_{24}$. Moreover, instead of $\nu_{\mu}$ and $\nu_{\tau}$, one can consider the linear combinations

$$
\left(\begin{array}{c}
\nu_{a} \\
\nu_{b}
\end{array}\right)=\left(\begin{array}{cc}
-\sin \vartheta & -\cos \vartheta \\
\cos \vartheta & -\sin \vartheta
\end{array}\right)\left(\begin{array}{cc}
\sin \vartheta_{34} & \cos \vartheta_{34} \\
\cos \vartheta_{34} & -\sin \vartheta_{34}
\end{array}\right)\left(\begin{array}{c}
\nu_{\mu} \\
\nu_{\tau}
\end{array}\right),
$$

with

$$
\tan \vartheta=\frac{\sin \vartheta_{24}}{\tan \vartheta_{23}}
$$

The mixing of $\nu_{a}$ and $\nu_{b}$ with $\nu_{1}$ and $\nu_{2}$ is given by

$$
\begin{gathered}
U_{a 1}=-s_{12} \sqrt{1-c_{23}^{2} c_{24}^{2}}, \quad U_{a 2}=c_{12} \sqrt{1-c_{23}^{2} c_{24}^{2}}, \\
U_{b 1}=U_{b 2}=0 .
\end{gathered}
$$

Therefore, the oscillations of solar neutrinos depend only on $\vartheta_{12}$ and the product $\cos \vartheta_{23} \cos \vartheta_{24}$. If $\cos \vartheta_{23} \cos \vartheta_{24}$ $\neq 1$, solar $\nu_{e}$ 's can transform in the linear combination $\nu_{a}$ of active $\nu_{\mu}$ and $\nu_{\tau}$. We distinguish the following limiting cases: If $\cos \vartheta_{23} \cos \vartheta_{24}=0$ then $U_{s 1}=U_{s 2}=0, U_{a 1}=$ $-\sin \vartheta_{12}, U_{a 2}=\cos \vartheta_{12}$, corresponding to the limit of pure two-generation $\nu_{e} \rightarrow \nu_{a}$ transitions; if $\cos \vartheta_{23} \cos \vartheta_{24}=1$ then $U_{s 1}=-\sin \vartheta_{12}, U_{s 2}=\cos \vartheta_{12}$ and $U_{a 1}=U_{a 2}=0$ and we have the limit of pure two-generation $\nu_{e} \rightarrow \nu_{s}$ transitions.

Since the mixing of $\nu_{e}$ with $\nu_{1}$ and $\nu_{2}$ is equal to the mixing in the case of two generations (with the mixing angle $\vartheta_{12}$ ), the mixing of $\nu_{s}$ with $\nu_{1}$ and $\nu_{2}$ is equal to the one in the case of two generations times $\cos \vartheta_{23} \cos \vartheta_{24}$ and the mixing of $\nu_{a}$ with $\nu_{1}$ and $\nu_{2}$ is equal to the one in the case of two generations times $\sqrt{1-\cos ^{2} \vartheta_{23} \cos ^{2} \vartheta_{24}}$, it is clear that in the general case of simultaneous $\nu_{e} \rightarrow \nu_{s}$ and $\nu_{e} \rightarrow \nu_{a}$ oscillations the corresponding probabilities are given by

$$
\begin{gathered}
P_{\nu_{e} \rightarrow \nu_{s}}^{\text {Sun }}=\cos ^{2} \vartheta_{23} \cos ^{2} \vartheta_{24}\left(1-P_{\nu_{e} \rightarrow \nu_{e}}^{\text {Sun }}\right), \\
P_{\nu_{e} \rightarrow \nu_{a}}^{\text {Sun }}=\left(1-\cos ^{2} \vartheta_{23} \cos ^{2} \vartheta_{24}\right)\left(1-P_{\nu_{e} \rightarrow \nu_{e}}^{\text {Sun }}\right) .
\end{gathered}
$$

These expressions satisfy the relation of probability conservation $P_{\nu_{e} \rightarrow v_{e}}^{\text {Sun }}+P_{v_{e} \rightarrow \nu_{s}}^{\text {Sun }}+P_{\nu_{e} \rightarrow \nu_{a}}^{\text {Sun }}=1$.

If $\Delta m_{21}^{2}$ is in the MSW region $\left(10^{-8} \mathrm{eV}^{2} \lesssim \Delta m_{21}^{2} \lesssim 3\right.$ $\times 10^{-4} \mathrm{eV}^{2}$ ), the survival probabilities of solar $\nu_{e}$ 's is given by [22]

$$
P_{\nu_{e} \rightarrow \nu_{e}}^{\text {Sun }}=\frac{1}{2}+\left(\frac{1}{2}-P_{c}\right) \cos 2 \vartheta_{12} \cos 2 \vartheta_{12}^{M}
$$

Here the angle $\vartheta_{12}^{M}$ is the effective mixing angle in matter corresponding to the vacuum mixing angle $\vartheta_{12}$ and given by

$$
\tan 2 \vartheta_{12}^{M}=\frac{\tan 2 \vartheta_{12}}{1-A / \Delta m_{21}^{2} \cos 2 \vartheta_{12}}
$$

with

$$
A \equiv A_{C C}+\cos ^{2} \vartheta_{23} \cos ^{2} \vartheta_{24} A_{N C}
$$

The quantities $A_{C C}$ and $A_{N C}$ describe the matter effects and are given by

$$
A_{C C}=2 \sqrt{2} G_{F} E N_{e}, \quad A_{N C}=-\sqrt{2} G_{F} E N_{n},
$$

where $N_{e}$ and $N_{n}$ are, respectively, the number densities of electrons and neutrons in the medium, $E$ is the neutrino energy, and $G_{F}$ is the Fermi constant. The effective mixing angle $\vartheta_{12}^{M}$ in Eqs. (2.12) and (2.10) must be evaluated at the point of neutrino production inside of the Sun. The quantity $P_{c}$ in Eq. (2.12) is the crossing probability given by the usual two-generation formula (see [25]) and the replacement of the two-generation expression for $A$ with that given in Eq. (2.14).

During the night solar neutrinos cross the Earth before reaching the detector and regeneration of $\nu_{e}$ 's is possible [23]. In the four-neutrino schemes under consideration, the probabilities of $\nu_{e} \rightarrow \nu_{e}$ and $\nu_{e} \rightarrow \nu_{s}$ transitions after crossing the Earth are given by [22] 


$$
\begin{gathered}
P_{\nu_{e} \rightarrow \nu_{e}}^{\text {Sun }+ \text { Earth }}=P_{\nu_{e} \rightarrow \nu_{e}}^{\text {Sun }}+\frac{\left(1-2 P_{\nu_{e} \rightarrow \nu_{e}}^{\text {Sun }}\right)\left(P_{\nu_{2} \rightarrow \nu_{e}}^{\text {Earth }}-\sin ^{2} \vartheta_{12}\right)}{\cos 2 \vartheta_{12}}, \\
P_{\nu_{e} \rightarrow \nu_{s}}^{\text {Sun+Earth }}=P_{\nu_{e} \rightarrow \nu_{s}}^{\text {Sun }}+\frac{\left(2 P_{\nu_{e} \rightarrow \nu_{s}}^{\text {Sun }}-\cos ^{2} \vartheta_{23} \cos ^{2} \vartheta_{24}\right)\left(P_{\nu_{2} \rightarrow \nu_{s}}^{\text {Earth }}-\cos ^{2} \vartheta_{12} \cos ^{2} \vartheta_{23} \cos ^{2} \vartheta_{24}\right)}{\cos 2 \vartheta_{12} \cos ^{2} \vartheta_{23} \cos ^{2} \vartheta_{24}} .
\end{gathered}
$$

The probability of $\nu_{e} \rightarrow \nu_{a}$ transitions is given by the conservation of probability: $\quad P_{\nu_{e} \rightarrow \nu_{a}}^{\text {Sun+Earth }}=1-P_{\nu_{e} \rightarrow \nu_{e}}^{\text {Sun+Earth }}$ $-P_{\nu_{e} \rightarrow \nu_{s}}^{\text {Sun+Earth }}$.

The probabilities $P_{\nu_{2} \rightarrow \nu_{e}}^{\text {Earth }}$ and $P_{\nu_{2} \rightarrow \nu_{s}}^{\text {Earth }}$ in Eqs. (2.16) and (2.17) can be calculated by integrating numerically the differential equation that describes the evolution of neutrino flavors in the Earth (see [22]) or by using the analytical solution assuming a step-function profile of the Earth matter density (see [24]) . However, we notice that the probabilities $P_{\nu_{2} \rightarrow \nu_{e}}^{\text {Earth }}$ and $P_{\nu_{2} \rightarrow \nu_{s}}^{\text {Earth }}$ are not independent, because, as shown in the Appendix, they are related by

$$
P_{\nu_{2} \rightarrow \nu_{s}}^{\text {Earth }}=\cos ^{2} \vartheta_{23} \cos ^{2} \vartheta_{24}\left(1-P_{\nu_{2} \rightarrow \nu_{e}}^{\text {Earth }}\right) \text {. }
$$

Therefore, in the analysis of solar neutrino data we need to calculate only $P_{\nu_{2} \rightarrow \nu_{e}}^{\text {Earth }}$.

If $\Delta m_{21}^{2}$ is in the range of the vacuum oscillation solution of the solar neutrino problem $\left(10^{-11} \mathrm{eV}^{2} \leq \Delta m_{21}^{2}\right.$ $\leqslant 10^{-9} \mathrm{eV}^{2}$ ), the survival probability of solar $\nu_{e}$ is given by the two-generation formula

$$
P_{\nu_{e} \rightarrow \nu_{e}}^{\text {Sun }}=1-\sin ^{2} 2 \vartheta_{12} \sin ^{2} \frac{\Delta m_{21}^{2} L}{4 E}
$$

where $E$ is the neutrino energy and $L$ is the Sun-Earth distance, whose seasonal variations must be taken into account. In this case there is no matter effect during neutrino propagation in the Earth.

\section{DATA AND TECHNIQUES}

In order to study the possible values of neutrino masses and mixing for the oscillation solution of the solar neutrino problem, we have used data on the total event rates measured in the chlorine experiment at Homestake [5], in the two gallium experiments GALLEX and SAGE $[6,7]$ and in the water Cherenkov detectors Kamiokande and SuperKamiokande shown in Table I. Apart from the total event

TABLE I. Measured rates for the chlorine, gallium, Kamiokande, and Super-Kamiokande experiments.

\begin{tabular}{lcccc}
\hline \hline Experiment & Rate & Ref. & Units & $R_{i}^{\text {BP98 }}$ \\
\hline Homestake & $2.56 \pm 0.23$ & {$[5]$} & SNU & $7.8 \pm 1.1$ \\
GALLEX + SAGE & $72.3 \pm 5.6$ & {$[6,7]$} & SNU & $130 \pm 7$ \\
Kamiokande & $2.80 \pm 0.38$ & {$[8]$} & $10^{6} \mathrm{~cm}^{-2} \mathrm{~s}^{-1}$ & $5.2 \pm 0.9$ \\
Super-Kamiokande & $2.45 \pm 0.08$ & {$[10]$} & $10^{6} \mathrm{~cm}^{-2} \mathrm{~s}^{-1}$ & $5.2 \pm 0.9$ \\
\hline \hline
\end{tabular}

rates, we have in this last case the zenith angle distribution of the events and the electron recoil energy spectrum, all measured with their recent 825-day data sample [10]. Although, as discuss in Ref. [26] the inclusion of Kamiokande results does not affect the shape of the regions, because of the much larger precision of the Super-Kamiokande measurement, it is convenient to introduce it as in this way the number of degrees of freedom for the fit of the rates only is $4-3=1$ (instead of zero degrees of freedom), that allows the construction of a well-defined $\chi_{\min }^{2}$ confidence level.

For the calculation of the theoretical expectations we use the BP98 standard solar model of Ref. [27]. The general expression of the expected event rate in the presence of oscillations in experiment $i$ in the four-neutrino framework is given by $R_{i}^{\text {th }}$ :

$$
\begin{aligned}
R_{i}^{\mathrm{th}}= & \sum_{k=1,8} \phi_{k} \int d E_{\nu} \lambda_{k}\left(E_{\nu}\right) \times\left[\sigma_{e, i}\left(E_{\nu}\right)\left\langle P_{\nu_{e} \rightarrow \nu_{e}}\right\rangle+\sigma_{x, i}\left(E_{\nu}\right)\right. \\
& \left.\times\left(1-\left\langle P_{\nu_{e} \rightarrow \nu_{e}}\right\rangle-\left\langle P_{\nu_{e} \rightarrow \nu_{s}}\right\rangle\right)\right] .
\end{aligned}
$$

where $E_{\nu}$ is the neutrino energy, $\phi_{k}$ is the total neutrino flux, and $\lambda_{k}$ is the neutrino energy spectrum (normalized to 1 ) from the solar nuclear reaction $k$ with the normalization given in Ref. [27]. Here $\sigma_{e, i}\left(\sigma_{x, i}\right)$ is the $\nu_{e}\left(\nu_{x}, x=\mu, \tau\right)$ interaction cross section in the Standard Model with the target corresponding to experiment $i$. For the chlorine and Gallium experiments we use improved cross sections $\sigma_{\alpha, i}(E)$ $(\alpha=e, x)$ from Ref. [28]. For the Kamiokande and SuperKamiokande experiment we calculate the expected signal with the corrected cross section as explained below. $\left\langle P_{\nu_{e} \rightarrow \nu_{\alpha}}\right\rangle$ is the time-averaged $\nu_{e}$ survival probability. In case of MSW transitions $P_{\nu_{e} \rightarrow \nu_{e}}$ and $P_{\nu_{e} \rightarrow \nu_{s}}$ are given in Eqs. (2.16) and (2.17), respectively.

For vacuum oscillations we must include the effect of the Earth orbit eccentricity. The yearly averaged probability is obtained by averaging Eq. (2.19) with $L(t)=L_{0}[1$ $-\varepsilon \cos 2 \pi(t / T)]$ :

$$
\begin{aligned}
\left\langle P_{\nu_{e} \rightarrow v_{e}}\right\rangle= & \left\langle P_{\nu_{e} \rightarrow v_{e}}^{\text {Sun }}\right\rangle=2-\sin ^{2} 2 \vartheta_{12} \\
& \times\left[1-\cos \left(\frac{\Delta m_{21}^{2} L_{0}}{2 E}\right) J_{0}\left(\frac{\varepsilon \Delta m_{21}^{2} L_{0}}{2 E}\right)\right],
\end{aligned}
$$

where $\varepsilon$ is the orbit eccentricity (0.0167), $L_{0}$ is the average Earth orbit radius $\left(1.496 \times 10^{8} \mathrm{~km}\right)$ and $J_{0}(x)$ is the Bessel function. We have also included in the fit the experimental 
results from the Super-Kamiokande Collaboration on the zenith angle distribution of events taken on 5 night periods and the day averaged value, which we graphically reduced from Ref. [10]. For MSW oscillations we compute the expected event rate in the period $a$ in the presence of oscillations as,

$$
\begin{aligned}
R_{s k, a}^{t h}= & \frac{1}{\Delta \tau_{a}} \int_{\tau\left(\cos \Phi_{\min , a}\right)}^{\tau\left(\cos \Phi_{\max , a}\right)} d \tau \sum_{k=1,8} \phi_{k} \int d E_{\nu} \lambda_{k}\left(E_{\nu}\right) \\
& \times\left[\sigma_{e, s k}\left(E_{\nu}\right)\left\langle P_{\nu_{e} \rightarrow \nu_{e}}(\tau)\right\rangle+\sigma_{x, s k}\left(E_{\nu}\right)\right. \\
& \left.\times\left(1-\left\langle P_{\nu_{e} \rightarrow \nu_{e}}(\tau)\right\rangle-\left\langle P_{\nu_{e} \rightarrow \nu_{s}}(\tau)\right\rangle\right)\right],
\end{aligned}
$$

where $\tau$ measures the yearly averaged length of the period $a$ normalized to 1 , so $\Delta \tau_{a}=\tau\left(\cos \Phi_{\max , a}\right)-\tau\left(\cos \Phi_{\min , a}\right)$ $=0.500,0.086,0.091,0.113,0.111,0.099$ for the day and five night periods. Notice that for vacuum oscillations there is no matter effect during neutrino propagation in the Earth. In this case $R_{s k, a}^{\mathrm{th}}=R_{s k}^{\mathrm{th}}$, as given in Eq. (3.1). The SuperKamiokande Collaboration has also presented the results on the day-night variation in the form of a day-night asymmetry. Since the information included in the zenith angle dependence already contains the day-night asymmetry, we have not added the asymmetry as an independent observable in our fit.

The Super-Kamiokande Collaboration has also measured the recoil electron energy spectrum. In their published analysis [9] after 504 days of operation they present their results for energies above 6.5 MeV using the low-energy (LE) analysis in which the recoil energy spectrum is divided into 16 bins, 15 bins of $0.5 \mathrm{MeV}$ energy width and the last bin containing all events with energy in the range $14-20 \mathrm{MeV}$. Below $6.5 \mathrm{MeV}$ the background of the LE analysis increases very fast as the energy decreases. Super-Kamiokande has designed a new super low-energy (SLE) analysis in order to reject this background more efficiently so as to be able to lower their threshold down to $5.5 \mathrm{MeV}$. In their 825-day data [10] they have used the SLE method and they present results for two additional bins with energies between 5.5 and 6.5 $\mathrm{MeV}$. In our study we use the experimental results from the Super-Kamiokande Collaboration on the recoil electron spectrum divided in 18 energy bins, including the results from the LE analysis for the 16 bins above $6.5 \mathrm{MeV}$ and the results from the SLE analysis for the two low-energy bins below $6.5 \mathrm{MeV}$. The general expression of the expected rate in a bin in the presence of oscillations $R^{\text {th }}$, is similar to that in Eq. (3.1), with the substitution of the cross sections with the corresponding differential cross sections folded with the finite energy resolution function of the detector and integrated over the electron recoil energy interval of the bin, $T_{\min } \leqslant T \leqslant T_{\max }$ :

$$
\begin{aligned}
\sigma_{\alpha, s k}\left(E_{\nu}\right)= & \int_{T_{\min }}^{T_{\max }} d T \int_{0}^{E_{\nu} /\left(1+m_{e} / 2 E_{\nu}\right)} d T^{\prime} \operatorname{Res}\left(T, T^{\prime}\right) \\
& \times \frac{d \sigma_{\alpha, s k}\left(E_{\nu}, T^{\prime}\right)}{d T^{\prime}} .
\end{aligned}
$$

The resolution function $\operatorname{Res}\left(T, T^{\prime}\right)$ is of the form $[9,29]$ :

$$
\begin{aligned}
\operatorname{Res}\left(T, T^{\prime}\right)= & \frac{1}{\sqrt{2 \pi}\left[0.47 \sqrt{T^{\prime}(\mathrm{MeV})}\right]} \\
& \times \exp \left[-\frac{\left(T-T^{\prime}\right)^{2}}{0.44 T^{\prime}(\mathrm{MeV})}\right],
\end{aligned}
$$

and we take the differential cross section $d \sigma_{\alpha}\left(E_{\nu}, T^{\prime}\right) / d T^{\prime}$ from [30].

In the statistical treatment of all these data we perform a $\chi^{2}$ analysis for the different sets of data, following closely the analysis of Ref. [31] with the updated uncertainties given in Refs. [26-28], as discussed in Ref. [32]. We thus define a $\chi^{2}$ function for the three set of observables $\chi_{\text {rates }}^{2}, \chi_{\text {zenith }}^{2}$, and $\chi_{\text {spectrum }}^{2}$ where in both $\chi_{\text {zenith }}^{2}$ and $\chi_{\text {spectrum }}^{2}$ we allow for a free normalization in order to avoid double counting with the data on the total event rate which is already included in $\chi_{\text {rates }}^{2}$. In the combinations of observables we define the $\chi^{2}$ of the combination as the sum of the different $\chi^{2}$ 's. In principle, such an analysis should be taken with a grain of salt as these pieces of information are not fully independent; in fact, they are just different projections of the double differential spectrum of events as a function of time and energy. Thus, in our combination we are neglecting possible correlations between the uncertainties in the energy and time dependence of the event rates.

\section{RESULTS}

As explained in Sec. II, for the mass scales invoked in the explanation of the atmospheric and LSND data and after imposing the strong constraints from the Bugey [33] and CHOOZ [34] reactor experiments, the relevant parameter space for solar neutrino oscillations in the framework of four-neutrino mixing is a three-dimensional space in the variables $\Delta m_{21}^{2}, \quad \vartheta_{12}$ and $\cos ^{2}\left(\vartheta_{23}\right) \cos ^{2}\left(\vartheta_{24}\right) \equiv c_{23}^{2} c_{24}^{2}$. As shown in Sec. II, the case $c_{23}^{2} c_{24}^{2}=0$ corresponds to the usual two-neutrino oscillations $\nu_{e} \rightarrow \nu_{a}$ where $\nu_{a}$ is the admixture of $\nu_{\mu}$ and $\nu_{\tau}$ given in Eq. (2.7), thus an active neutrino. The other extreme case $c_{23}^{2} c_{24}^{2}=1$ corresponds to the usual twoneutrino oscillations of $\nu_{e}$ into a pure sterile neutrino.

In our choice of ordering the neutrino masses in the two schemes (2.1) the mass-squared difference $\Delta m_{21}^{2}$ is positive. The mixing angle $\vartheta_{12}$ can vary in the interval $0 \leqslant \vartheta_{12} \leqslant \pi / 2$. In the case of vacuum oscillations, the transition probabilities are symmetric under the change $\vartheta_{12} \rightarrow \pi / 2-\vartheta_{12}$ and each allowed value of $\sin ^{2}\left(2 \vartheta_{12}\right)$ corresponds to two allowed values of $\vartheta_{12}$. On the other hand, in the case of the MSW solutions the transition probabilities are not invariant under the change $\vartheta_{12} \rightarrow \pi / 2-\vartheta_{12}$ and resonant transitions are possible only for values of $\vartheta_{12}$ smaller than $\pi / 4$. In the analysis of the observable rates, we present the results in the common plot of $\sin ^{2}\left(2 \vartheta_{12}\right)$ due to the fact that the allowed region does not extend to $\vartheta_{12}>\pi / 4$. But, when we include the rest of observables we remark that this is not the case, and we present the results as a function of $\sin ^{2}\left(\vartheta_{12}\right)$ (see [35]) showing that there is a portion of the space of parameters in the second octant of $\vartheta_{12}$ allowed at 99\% C.L. This enlarged 


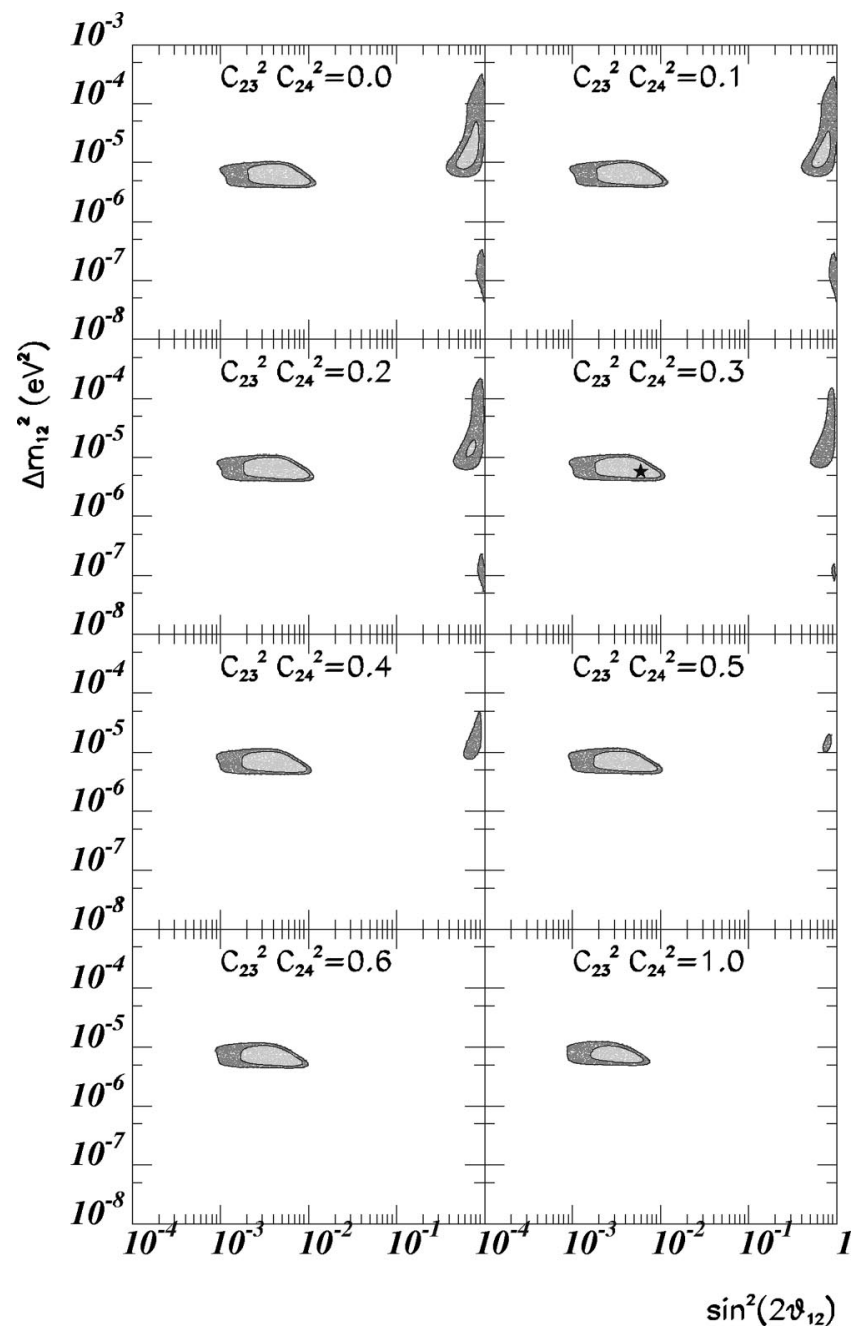

FIG. 1. Allowed regions in $\Delta m_{21}^{2}$ and $\sin ^{2}\left(2 \vartheta_{12}\right)$ for the MSW four-neutrino oscillations from the measurements of the total event rates at chlorine, gallium, Kamiokande, and Super-Kamiokande (825-day data sample). The different panels represent the allowed regions at $99 \%$ (darker) and $90 \%$ C.L. (lighter) obtained as sections for fixed values of the mixing angles $c_{13}^{2} c_{23}^{2}$ of the threedimensional volume defined by $\chi^{2}-\chi_{\min }^{2}=6.25$ (90\%), 11.36 (99\%). The best-fit point in the three-parameter space is plotted as a star.

parameter space is also used in Ref. [26].

We first present the results of the allowed regions in the three-parameter space for the different combination of observables. In building these regions, for a given set of observables, we compute for any point in the parameter space of four-neutrino oscillations the expected values of the observables and with those and the corresponding uncertainties we construct the function $\chi^{2}\left(\Delta m_{12}^{2}, \vartheta_{12}, c_{23}^{2} c_{24}^{2}\right)_{o b s}$. We find its minimum in the full three-dimensional space considering as a unique framework both MSW and vacuum oscillations. The allowed regions for a given C.L. are then defined as the set of points satisfying the condition:

$$
\chi^{2}\left(\Delta m_{12}^{2}, \vartheta_{12}, c_{23}^{2} c_{24}^{2}\right)_{o b s}-\chi_{m i n, o b s}^{2} \leqslant \Delta \chi^{2}(\text { C.L., } 3 \text { dof }),
$$

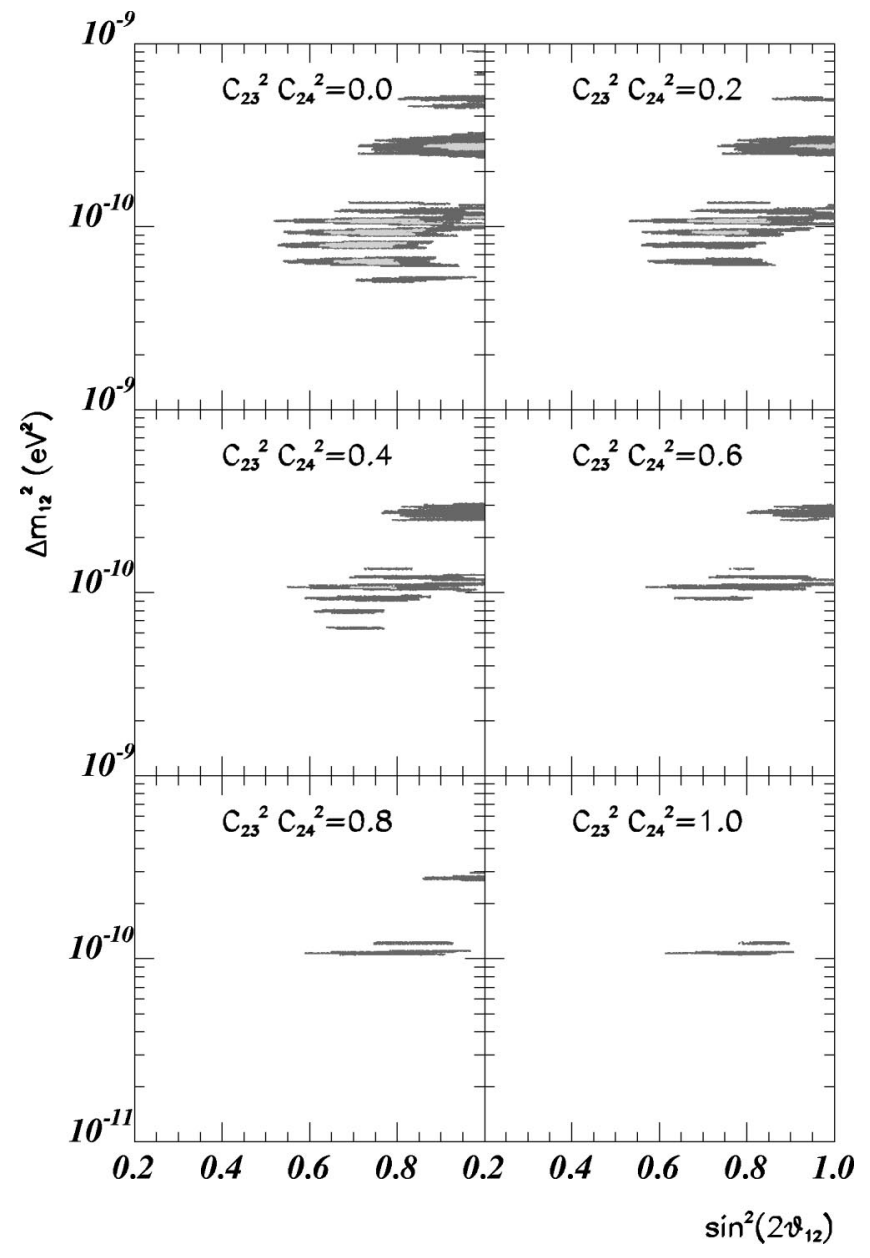

FIG. 2. Allowed regions in $\Delta m_{21}^{2}$ and $\sin ^{2}\left(2 \vartheta_{12}\right)$ for the vacuum four-neutrino oscillations from the measurements of the total event rates at chlorine, gallium, Kamiokande, and Super-Kamiokande (825-day data sample). The different panels represent the allowed regions at $99 \%$ (darker) and $90 \%$ C.L. (lighter) obtained as sections for fixed values of the mixing angles $c_{13}^{2} c_{23}^{2}$ of the threedimensional volume defined by $\chi^{2}-\chi_{\min }^{2}=6.25$ (90\%), 11.36 $(99 \%)$ where $\chi_{\min }^{2}$ is in the MSW region.

where, for instance, $\Delta \chi^{2}$ (C.L., 3 dof $)=6.25,7.83$, and 11.36 for C.L. $=90,95$, and 99\%, respectively. In Figs. 1-7 we plot the sections of such volume in the plane $\left[\Delta m_{21}^{2}, \sin ^{2}\left(2 \vartheta_{12}\right)\right]$ or $\left[\Delta m_{21}^{2}, \sin ^{2}\left(\vartheta_{12}\right)\right]$ for different values of $c_{23}^{2} c_{24}^{2}$.

Figures 1 and 2 show the results of the fit to the observed total rates only. We find that both at 90 and 99\% C.L., the three-dimensional allowed volume is composed of three separated three-dimensional regions in the MSW sector of the parameter space (Fig. 1), which we denote as SMA, LMA, and LOW solutions following the usual two-neutrino oscillation picture and a "tower" of regions in the vacuum oscillations sector (Fig. 2). The values of the minimum of the $\chi^{2}$ in the different regions are given in Table II. The global minimum used in the construction of the volumes lies in the SMA region and for a nonvanishing value of $c_{23}^{2} c_{24}^{2}=0.3$, although, as can be seen in the first panel in Fig. 8, this is of very little statistical significance as $\Delta \chi^{2}$ for the SMA solu- 


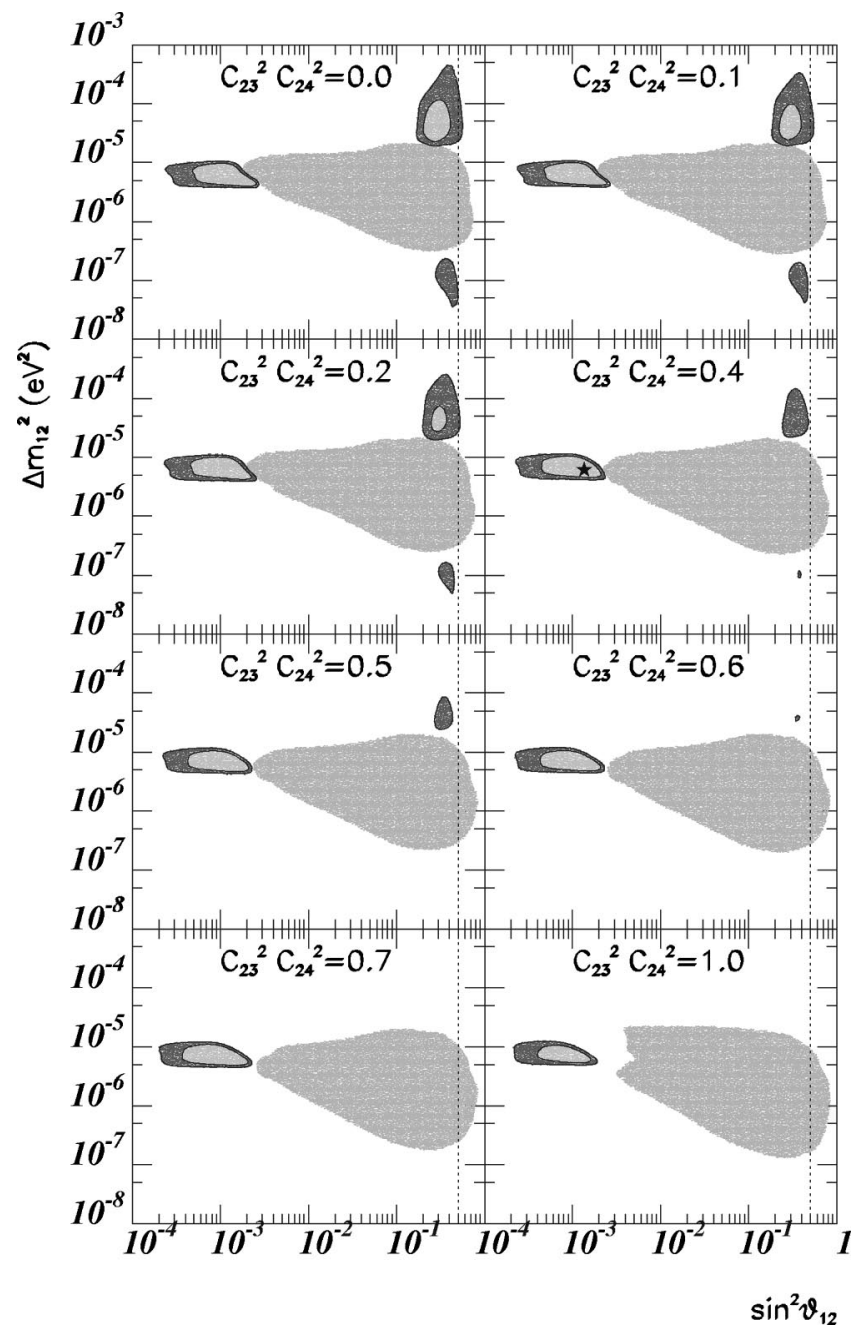

FIG. 3. Allowed regions in $\Delta m_{21}^{2}$ and $\sin ^{2} \vartheta_{12}$ for the MSW four-neutrino oscillations from the measurements of the event rates and the Super-Kamiokande zenith angular dependence data. The shadowed area represents the excluded region at $99 \%$ C.L. from the zenith angular data.

tion is very mildly dependent on $c_{23}^{2} c_{24}^{2}\left(\Delta \chi^{2} \lesssim 0.5\right.$ for $\left.c_{23}^{2} c_{24}^{2} \lesssim 0.5\right)$.

As seen in Fig. 1, the SMA region is always a valid solution for any value of $c_{23}^{2} c_{24}^{2}$. This is expected as in the two-neutrino oscillation picture this solution holds both for pure active-active and pure active-sterile oscillations. ${ }^{1}$ On the other hand, both the LMA and LOW solutions disappear for a value of the mixing $c_{23}^{2} c_{24}^{2} \geq 0.5(0.3)$. Unlike active neutrinos which lead to events in the water Cherenkov de-

\footnotetext{
${ }^{1}$ Notice, however, that the statistical analysis is different: in the two-neutrino picture the pure active-active and active-sterile cases are analyzed separately, whereas in the four-neutrino picture they are taken into account simultaneously in a consistent scheme that allows us to calculate the allowed regions with the prescription given in Eq. (4.1). We think that the agreement between the results of the analyses with two and four neutrinos indicate that the physical conclusions are quite robust.
}

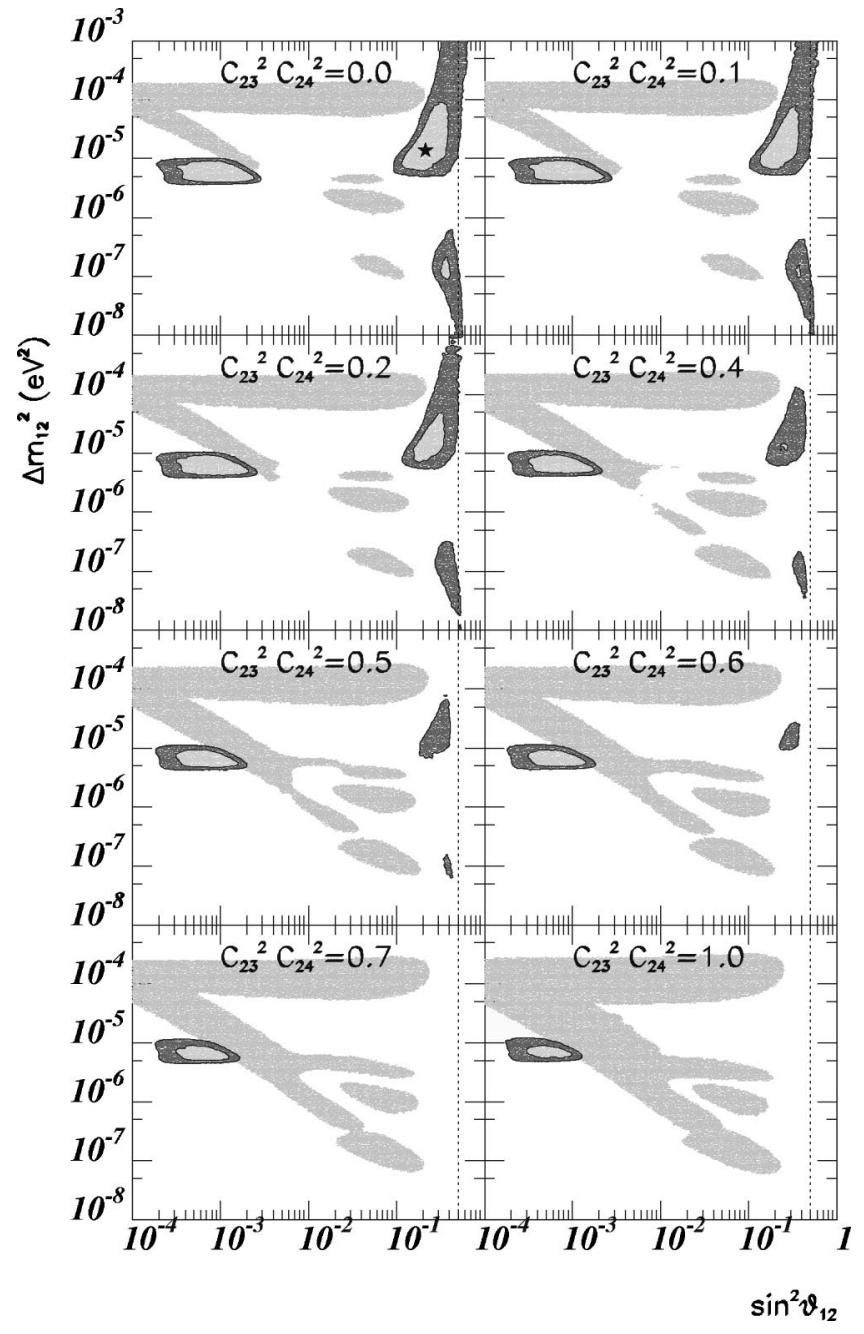

FIG. 4. Same as Fig. 3 but for the measurements of the event rates and the Super-Kamiokande recoil electron energy spectrum. The shadowed area represents the excluded region at 99\% C.L. from the energy spectrum.

tectors by interacting via neutral current with the electrons, sterile neutrinos do not contribute to the Kamiokande and Super-Kamiokande event rates. Therefore, a larger survival probability for ${ }^{8} B$ neutrinos is needed to accommodate the measured rate. As a consequence a larger contribution from ${ }^{8} B$ neutrinos to the chlorine and gallium experiments is expected, so that the small measured rate in chlorine can only be accommodated if no ${ }^{7} \mathrm{Be}$ neutrinos are present in the flux. This is only possible in the SMA solution region, since in the LMA and LOW regions the suppression of ${ }^{7} \mathrm{Be}$ neutrinos is not enough.

In Table III we give the maximum values of $c_{23}^{2} c_{24}^{2}$ for which the different solutions are allowed at the 90 and $99 \%$ C.L. according to different statistical criteria which we discuss below. In Fig. 2 we plot the corresponding sections in the vacuum oscillation sector. As seen in the figure, as $c_{23}^{2} c_{24}^{2}$ grows, the vacuum oscillation solution becomes more restricted in the allowed values of mass splittings until becoming a narrow band at $\Delta m_{21}^{2} \sim 10^{-10} \mathrm{eV}^{2}$ for the pure sterile case. 


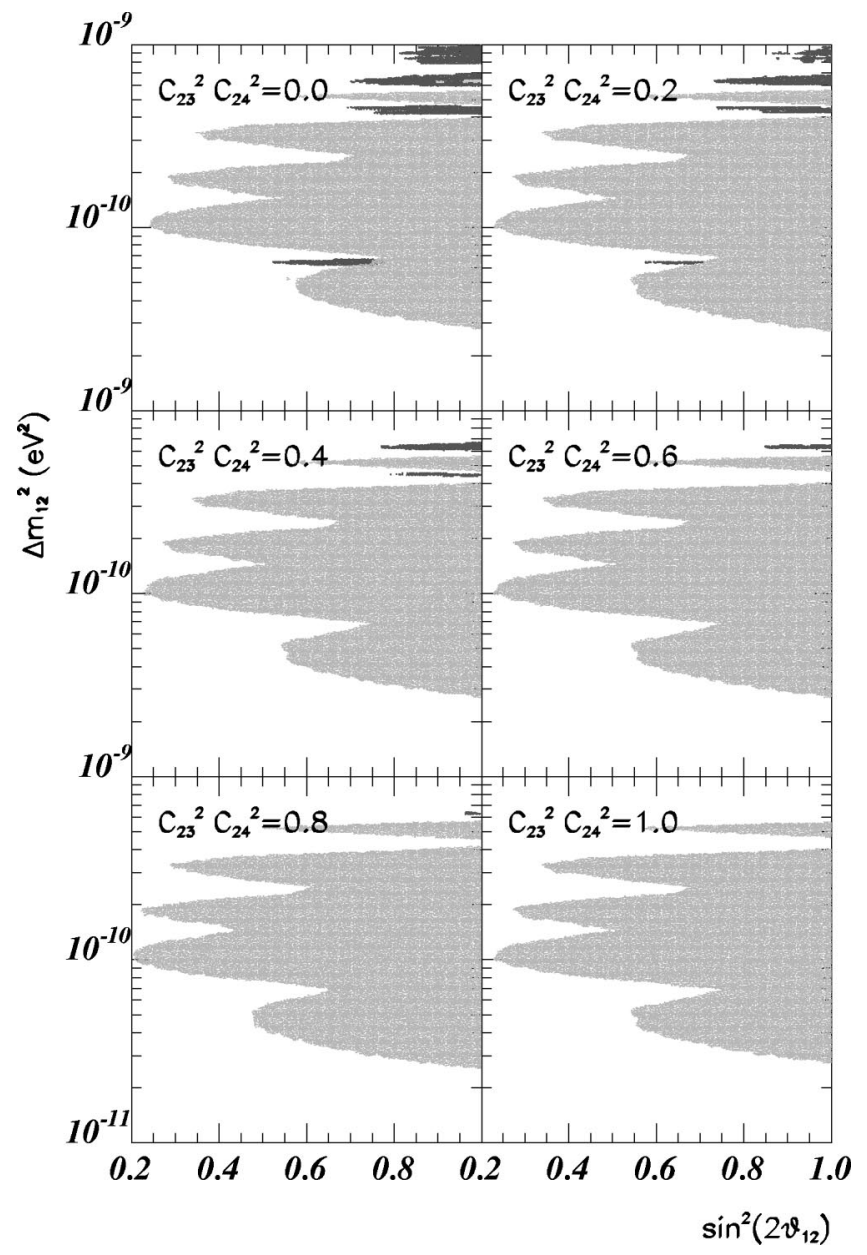

FIG. 5. Same as Fig. 2 but for the measurement of the event rates and the Super-Kamiokande recoil electron energy spectrum. The shadowed area represents the excluded region at 99\% C.L. from the energy spectrum.

Figure 3 shows the regions allowed by the fit of both total rates and the Super-Kamiokande zenith angular distribution in the MSW sector of the parameter space. In the vacuum oscillation section no day-night variation is expected. Also plotted is the excluded region at 99\% C.L. from the zenith angular measurement. This exclusion volume is built as the corresponding three-degrees-of-freedom region for the $\chi^{2}$ of the zenith angular data with respect to the minimum value $\chi_{\text {min,zen }}^{2}=0.8$ which occurs at $\Delta m_{21}^{2}=2.7 \times 10^{-6} \mathrm{eV}^{2}$, $\sin ^{2}\left(\vartheta_{12}\right)=0.85$, and $c_{23}^{2} c_{24}^{2}=0.0$. We remark that this minimum is placed in the second octant and this was not included in past analysis of two-flavor MSW solutions although it leads to little effect in the final results of the allowed regions. As seen in the figure and also in Table III, the main effect of the inclusion of the day-night variation data is to cut down the lower part of the LMA region and to push towards slightly higher values the maximum $c_{23}^{2} c_{24}^{2}$ for which the LMA and the LOW solutions are still valid.

In Figs. 4 and 5 we plot the regions allowed by the fit of both total rates and the Super-Kamiokande energy spectrum. Also plotted is the excluded region at 99\% C.L. from the spectrum data which is obtained as the corresponding three-

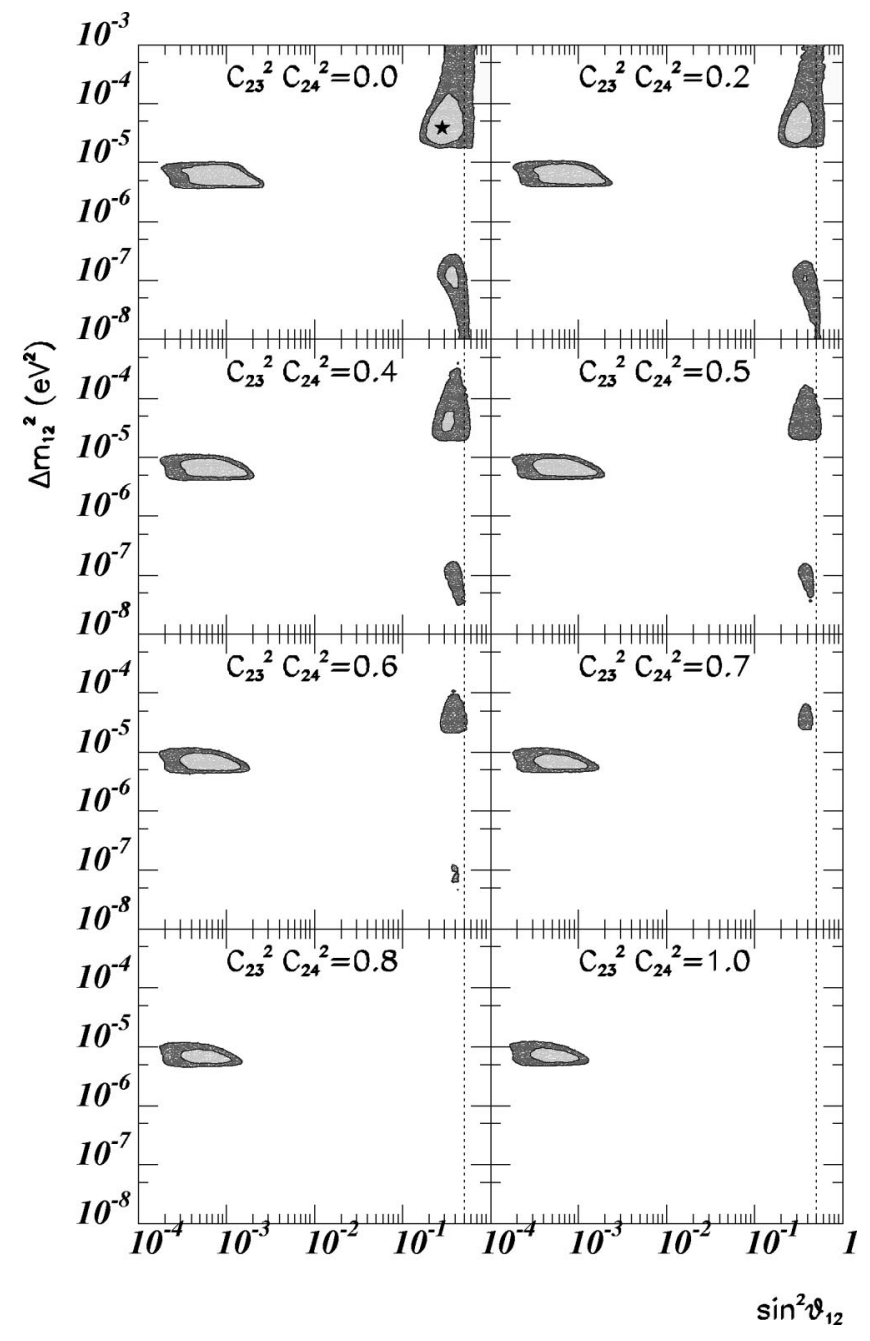

FIG. 6. Results of the global analysis for the allowed regions in $\Delta m_{21}^{2}$ and $\sin ^{2} \vartheta_{12}$ for the MSW four-neutrino oscillations. The light (dark) regions are allowed at $90 \%$ C.L. (99\% C.L.).

degrees-of-freedom region for the $\chi^{2}$ of the spectrum data with respect to the minimum value $\chi_{\text {min,spec }}^{2}=15.1$, which occurs in the vacuum solution sector at $\Delta m_{21}^{2}=6.3$ $\times 10^{-10} \mathrm{eV}^{2}$ and $\sin ^{2}\left(2 \vartheta_{12}\right)=1$, and it is almost independent of $c_{23}^{2} c_{24}^{2}$. As seen in the figure and also in Table III, the main effect of the inclusion of the spectrum data in the MSW regions is also to push towards slightly higher values the maximum $c_{23}^{2} c_{24}^{2}$ for which the LMA and the LOW solutions are still valid. Figure 4 shows that the LMA region at $99 \%$ C.L. extends to high values of $\Delta m_{21}^{2}$, even above $10^{-3} \mathrm{eV}^{2}$ for $c_{23}^{2} c_{24}^{2} \leq 0.1$. Since the atmospheric mass squared difference $\Delta m_{\mathrm{atm}}^{2}$ lies between $10^{-3}$ and $10^{-2} \mathrm{eV}^{2}$ (see [36]), one may wonder if the solar and atmospheric mass squared differences may coincide and three massive neutrinos may be enough for the explanation of solar, atmospheric, and LSND data. The answer to this question is negative, because in the high- $\Delta m_{21}^{2}$ part of the $99 \%$ C.L. LMA region the mixing angle $\theta_{21}$ is large, $0.3 \lessgtr \sin ^{2}\left(\theta_{21}\right) \lessgtr 0.7$, and in this case disappearance of $\bar{\nu}_{e}$ 's should be observed in long-baseline reactor experiments, contrary to the results of the CHOOZ [34] experiment. In other words, the results of the $\mathrm{CHOOZ}$ ex- 


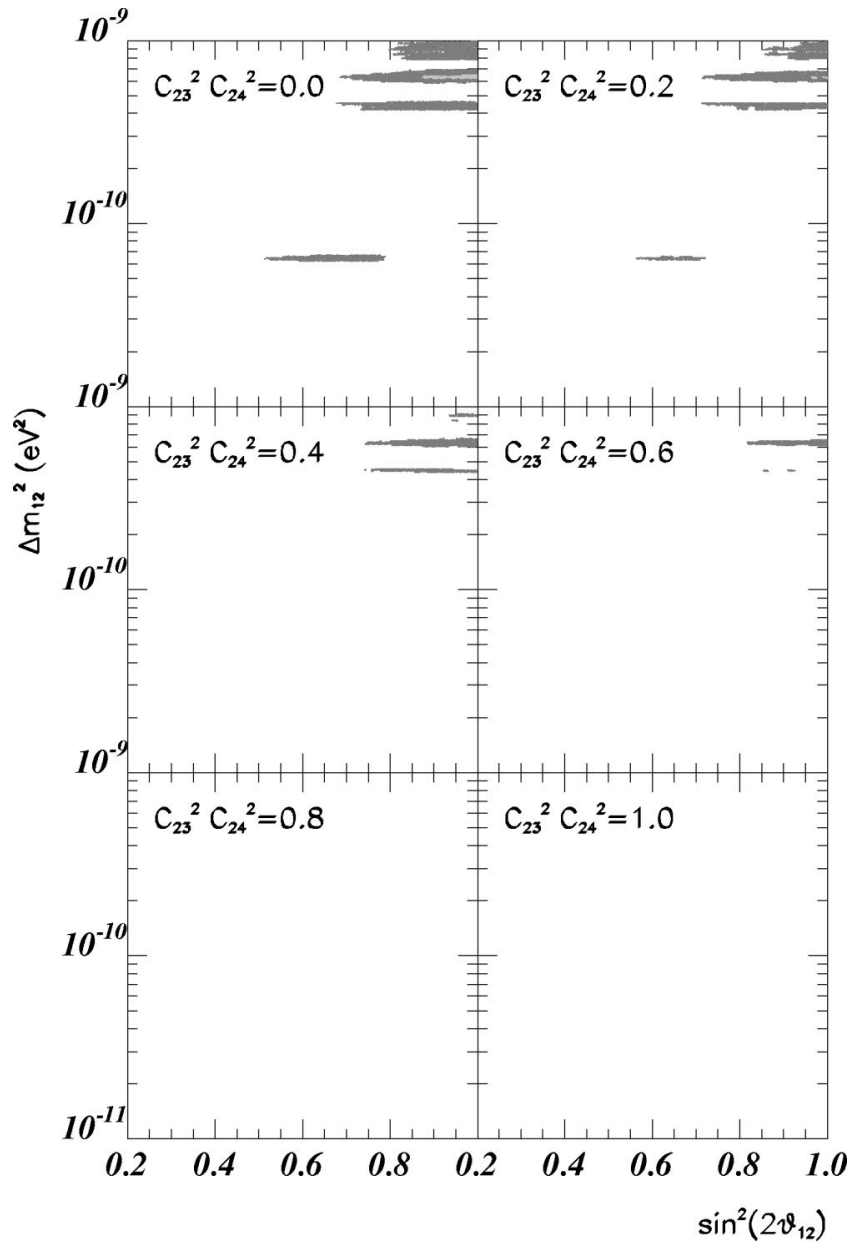

FIG. 7. Results of the global analysis for the allowed regions in $\Delta m_{21}^{2}$ and $\sin ^{2}\left(2 \vartheta_{12}\right)$ for the vacuum four-neutrino oscillations. The light (dark) regions are allowed at $90 \%$ C.L. (99\% C.L.).
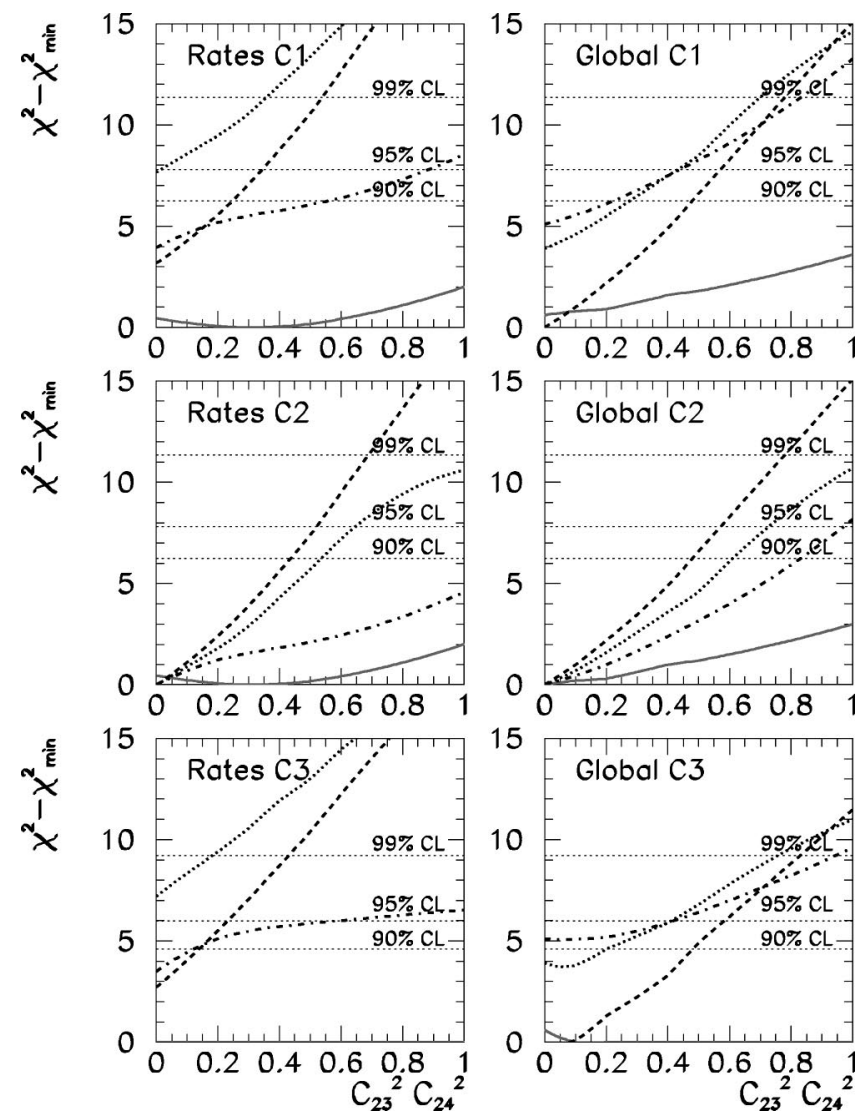

FIG. 8. $\Delta \chi^{2}$ as a function of the mixing parameter $c_{23}^{2} c_{24}^{2}$ for the different solutions SMA (full line), LMA (dashed), LOW (dotted), and vacuum (dot-dashed) from the analysis of the rates only right panels and from the analysis of the full data set (right panels). Each row represents the value for the different statistical criteria $\mathrm{C} 1, \mathrm{C} 2$, and $\mathrm{C} 3$ as defined in the text. The dotted horizontal lines correspond to the $90,95,99 \%$ C.L. limits for each criteria.

TABLE II. Best-fit points and the corresponding probabilities for the different solutions to the solar neutrino deficit and for different combinations of observables.

\begin{tabular}{|c|c|c|c|c|c|}
\hline & & Rates & Rates + zenith & Rates + spectrum & Global \\
\hline & $\Delta m^{2}$ & $5.6 \times 10^{-6}$ & $6.2 \times 10^{-6}$ & $5.2 \times 10^{-6}$ & $5.2 \times 10^{-6}$ \\
\hline \multirow[t]{4}{*}{ SMA } & $\sin ^{2}(2 \vartheta)$ & $6.0 \times 10^{-3}$ & $5.4 \times 10^{-3}$ & $4.7 \times 10^{-3}$ & $4.7 \times 10^{-3}$ \\
\hline & $c_{23}^{2} c_{24}^{2}$ & 0.3 & 0.4 & 0.0 & 0.0 \\
\hline & $\chi_{\min }^{2}(\operatorname{Prob} \%)$ & $0.55(45)$ & $6.2(40)$ & $23.9(16)$ & $29.7(16)$ \\
\hline & $\Delta m^{2}$ & $1.4 \times 10^{-5}$ & $4.2 \times 10^{-5}$ & $1.4 \times 10^{-5}$ & $3.9 \times 10^{-5}$ \\
\hline \multirow[t]{4}{*}{ LMA } & $\sin ^{2}(2 \vartheta)$ & 0.68 & 0.81 & 0.68 & 0.78 \\
\hline & $c_{23}^{2} c_{24}^{2}$ & 0.0 & 0.0 & 0.0 & 0.0 \\
\hline & $\chi_{\min }^{2}(\operatorname{Prob} \%)$ & $3.80(5)$ & $8.6(20)$ & $23.1(19)$ & $29.1(18)$ \\
\hline & $\Delta m^{2}$ & $1.3 \times 10^{-7}$ & $1.1 \times 10^{-7}$ & $1.0 \times 10^{-7}$ & $1.0 \times 10^{-7}$ \\
\hline \multirow[t]{4}{*}{ LOW } & $\sin ^{2}(2 \vartheta)$ & 0.93 & 0.93 & 0.93 & 0.93 \\
\hline & $c_{23}^{2} c_{24}^{2}$ & 0.0 & 0.0 & 0.0 & 0.0 \\
\hline & $\chi_{\min }^{2}(\operatorname{Prob} \%)$ & $8.3(0.4)$ & $13.6(3.4)$ & $27.9(6.4)$ & $33.0(8.1)$ \\
\hline & $\Delta m^{2}$ & $9.1 \times 10^{-11}$ & $9.1 \times 10^{-11}$ & $4.5 \times 10^{-10}$ & $4.4 \times 10^{-10}$ \\
\hline \multirow[t]{3}{*}{ Vacuum } & $\sin ^{2}(2 \vartheta)$ & 0.78 & 0.78 & 0.9 & 0.9 \\
\hline & $c_{23}^{2} c_{24}^{2}$ & 0.0 & 0.0 & 0.0 & 0.0 \\
\hline & $\chi_{\min }^{2}(\operatorname{Prob} \%)$ & $4.5(3.4)$ & $9.9(13)$ & $28.8(5.1)$ & $34.3(6.1)$ \\
\hline
\end{tabular}


TABLE III. Maximum allowed value of $\cos _{23}^{2} \cos _{24}^{2}$ at $90 \%$ and $99 \%$ C.L. for the different solutions to the solar neutrino problem with the different statistical criteria. SMA is allowed at $90 \%$ for all the range of $\cos _{23}^{2} \cos _{24}^{2}$.

\begin{tabular}{|c|c|c|c|c|}
\hline Observ. & Crit. & LMA 90 (99) & LOW 90 (99) & VAC 90 (99) \\
\hline & $\mathrm{C} 1$ & $0.25(0.54)$ & $-(0.36)$ & $0.56(1.0)$ \\
\hline \multirow[t]{3}{*}{ Rates } & $\mathrm{C} 2$ & $0.44(0.69)$ & $0.54(1.00)$ & $1.0(1.0)$ \\
\hline & $\mathrm{C} 3$ & $0.14(0.43)$ & $-(0.18)$ & $0.12(1.0)$ \\
\hline & $\mathrm{C} 1$ & $0.30(0.61)$ & $-(0.45)$ & $0.61(1.0)$ \\
\hline \multirow[t]{3}{*}{ Rates +SK Zenith } & $\mathrm{C} 2$ & $0.45(0.74)$ & $0.59(0.90)$ & $1.0(1.0)$ \\
\hline & $\mathrm{C} 3$ & $0.19(0.49)$ & $-(0.23)$ & $0.15(1.0)$ \\
\hline & $\mathrm{C} 1$ & $0.43(0.67)$ & $0.18(0.56)$ & $0.12(0.77)$ \\
\hline \multirow[t]{3}{*}{ Rates +SK Spect } & $\mathrm{C} 2$ & $0.43(0.67)$ & $0.54(1.0)$ & $0.83(1.0)$ \\
\hline & $\mathrm{C} 3$ & $0.45(0.72)$ & $-(0.57)$ & $-(0.93)$ \\
\hline & $\mathrm{C} 1$ & $0.48(0.78)$ & $0.28(0.70)$ & $0.22(0.83)$ \\
\hline \multirow[t]{2}{*}{ Global fit } & $\mathrm{C} 2$ & $0.48(0.78)$ & $0.61(1.0)$ & $0.83(1.0)$ \\
\hline & $\mathrm{C} 3$ & $0.48(0.83)$ & $0.20(0.76)$ & $-(0.95)$ \\
\hline
\end{tabular}

periment, that have not been taken into account in the present analysis, forbid the part of the $99 \%$ C.L. LMA region that extends above $\Delta m_{21}^{2} \simeq 10^{-3} \mathrm{eV}^{2}$. For this reason we cut the plots at this value. For the vacuum sector, once the spectrum data is included the higher $\Delta m_{21}^{2}$ are favored but we find no region at the $90 \%$ C.L. for any value of $c_{23}^{2} c_{24}^{2} \geq 0.2$ and at the $99 \%$ C.L. the region totally disappears for $c_{23}^{2} c_{24}^{2} \gtrsim 0.8$.

Figures 6 and 7 show the results from the global fit of the full set of data. The values of the minimum $\chi^{2}$ in the different regions are given in Table II. The global minimum used in the construction of the volumes lies in the LMA region and for vanishing $c_{23}^{2} c_{24}^{2}$ corresponding to pure $\nu_{e}$-active neutrino oscillations.

In Table III we give the maximum values of $c_{23}^{2} c_{24}^{2}$ for which the different solutions are allowed at the 90 and $99 \%$ C.L. according to different statistical criteria. The use of each criteria depends on the physics scenario to which the result of our analysis is to be applied.

Criterion 1 (C1). The maximum allowed value of the mixing $c_{23}^{2} c_{24}^{2}$ at a given C.L. for a given solution is defined as the value for which the corresponding region of the allowed three-dimensional volume defined as a 3-dof shift with respect to the global minimum in the full parameter space, disappears. In the first row in Fig. 8 we plot the values of $\Delta \chi^{2}$ defined in this way for the different solutions as a function of $c_{23}^{2} c_{24}^{2}$ for the fit of the total rates only (left panels) and for the global analysis (right panels). This criterion is the one used in building the regions in Figs. 1-7. It is applicable to models where no region of the parameter space MSW-SMA, MSW-LMA, MSW-LOW or vacuum is favored.

Criterion 2 (C2): The maximum allowed value of the mixing $c_{23}^{2} c_{24}^{2}$ at a given C.L. for a given solution is defined as the value for which the corresponding allowed threedimensional region defined as a 3-dof shift with respect to the local minimum in the corresponding region, disappears. In the second row in Fig. 8, we plot the values of $\Delta \chi^{2}$ defined in this way for the different solutions as a function of $c_{23}^{2} c_{24}^{2}$ for the fit to the total rates only (left panels) and for the global analysis (right panels). This criterion holds for models where only a certain solution, MSW-SMA, MSWLMA, MSW-LOW or vacuum is possible and it yields less restrictive limits.

Criterion 3 (C3): The maximum allowed value of the mixing $c_{23}^{2} c_{24}^{2}$ at a given C.L. is obtained calculating the two-dimensional allowed regions in the $\sin ^{2} \theta_{12}-\Delta m_{12}^{2}$ plane for each fixed value of $c_{23}^{2} c_{24}^{2}$. These allowed regions are defined through the 2-dof shift with respect to the global minimum in the plane $\sin ^{2} \theta_{12}-\Delta m_{12}^{2}$ (this is analogous to Criterion 1 for two parameters). For each solution, the maximum allowed value of $c_{23}^{2} c_{24}^{2}$ is that for which the corresponding two-dimensional region in the $\sin ^{2} \theta_{12}-\Delta m_{12}^{2}$ disappears. This criterion is the equivalent to the usual twoneutrino analysis but with $\nu_{e}$ oscillating into a state which is a given superposition of active and sterile neutrino. In the third row in Fig. 8 we plot the difference $\Delta \chi^{2}$ between the local minimum of $\chi^{2}$ for each solution and the global minimum in the plane $\sin ^{2} \theta_{12}-\Delta m_{12}^{2}$ as a function of $c_{23}^{2} c_{24}^{2}$. Notice that for the analysis of rates only the minimum in the plane $\sin ^{2} \theta_{12}$ and $\Delta m_{12}^{2}$ occurs always in the MSW-SMA region for any value of $c_{23}^{2} c_{24}^{2}$. Therefore, the curve for the MSW-SMA solution corresponds to the horizontal $\Delta \chi^{2}=0$ line and it is not shown. For the global analysis, when $c_{23}^{2} c_{24}^{2}<0.1$ the minimum in the plane occurs for the MSWLMA (dashed line) solution, while for $c_{23}^{2} c_{24}^{2}>0.1$ it moves to the MSW-SMA (full line). For this reason the curve for the MSW-SMA (MSW-LMA) solution is only seen for $c_{23}^{2} c_{24}^{2}<0.1(>0.1)$, while for $c_{23}^{2} c_{24}^{2}>0.1(<0.1)$ it coincides with the $\Delta \chi^{2}=0$ line. In general this criterion is applicable for models where the additional mixing $c_{23}^{2} c_{24}^{2}$ is fixed a priori to some value, so that the model in fact contains only two free parameters (if $c_{23}^{2} c_{24}^{2}$ is larger than the maximum allowed for a given solution, it means that that solution is not allowed in the specific model).

\section{SUMMARY AND DISCUSSION}

At present, the standard model assumption of massless neutrinos is under question due to the important results of 
underground experiments. Altogether they provide solid evidence for the existence of anomalies in the solar and atmospheric neutrino fluxes which could be accounted for in terms of neutrino oscillations $\nu_{e} \rightarrow \nu_{x}$ and $\nu_{\mu} \rightarrow \nu_{x}$, respectively. Together with these results there is also the indication of neutrino oscillations in the $\bar{\nu}_{\mu} \rightarrow \bar{\nu}_{e}$ channel obtained in the LSND experiment. All these experimental results can be accommodated in a single neutrino oscillation framework only if there are at least three different scales of neutrino mass-squared differences. The simplest way to open the possibility of incorporating the LSND scale in the solar and atmospheric neutrino scales is to invoke a sterile neutrino, i.e., one whose interaction with standard model particles is much weaker than the SM weak interaction, so that it does not affect the invisible $Z$ decay width, precisely measured at LEP. The sterile neutrino must also be light enough in order to participate in the oscillations involving the three active neutrinos. After imposing the present constraints from the negative searches at accelerator and reactor neutrino oscillation experiments, one is left with two possible mass patterns which can be included in a single four-neutrino framework as described in Sec. II.

In this paper we have performed an analysis of the neutrino oscillation solutions to the solar neutrino problem in the framework of four-neutrino mixing. We consider both transitions via the Mikheyev-Smirnov-Wolfenstein (MSW) mechanism as well as oscillations in vacuum. Where solar neutrinos are concerned, our formalism contains one additional parameter as compared to the pure two-neutrino case: $\cos ^{2}\left(\vartheta_{23}\right) \cos ^{2}\left(\vartheta_{24}\right)$, where $\vartheta_{23}$ and $\vartheta_{24}$ give the projections of the sterile neutrino into each of the two heavier states responsible for explanation of the atmospheric neutrino anomaly. In this way, the formalism permits transitions into active or sterile neutrinos controlled by the additional parameter and contains as limiting cases the pure $\nu_{e}$-active and $\nu_{e}$-sterile neutrino oscillations.

We have studied the evolution of the different solutions to the solar neutrino problem in this three-parameter space when the different sets of observables are included. In Figs. 1-7 we plot the sections of such volumes in the plane $\left[\Delta m_{21}^{2}, \sin ^{2}\left(2 \vartheta_{12}\right)\right]$ or $\left[\Delta m_{21}^{2}, \sin ^{2}\left(\vartheta_{12}\right)\right]$ for different values of $c_{23}^{2} c_{24}^{2}$. As a particularity, we also show that for MSW transitions there are solutions at $99 \%$ C.L. at $\vartheta_{12}$ mixing angles greater than $\pi / 4$ and that the best-fit point for the zenith angle distribution is in the second octant.

Our results show that the SMA region is always a valid solution for any value of $c_{23}^{2} c_{24}^{2}$. This is expected as in the two-neutrino oscillation picture this solution holds both for pure active-active and pure active-sterile oscillations. On the other hand, the LMA, LOW, and vacuum solutions become worse as the additional mixing $c_{23}^{2} c_{24}^{2}$ grows and they get to disappear for large values of the mixing. The main quantitative results of our analysis are summarized in Table III and Fig. 8 where we give the maximum values of $c_{23}^{2} c_{24}^{2}$ for which the different solutions are allowed at the 90 and $99 \%$ C.L. according to different statistical criteria which depend on the physics scenario to which the result of our analysis is to be applied.

\section{ACKNOWLEDGMENTS}

M.C.G.-G. is thankful to the CERN theory division for their kind hospitality during her visit. This work was supported by Spanish DGICYT under grant PB95-1077 and by the European Union TMR network ERBFMRXCT960090.

\section{APPENDIX: DERIVATION OF THE RELATION BETWEEN $\boldsymbol{P}_{\boldsymbol{\nu}_{2} \rightarrow \boldsymbol{\nu}_{e}}^{\text {Earth }}$ AND $\boldsymbol{P}_{\boldsymbol{\nu}_{2} \rightarrow \boldsymbol{\nu}_{s}}^{\text {Earth }}$}

In this appendix we derive the relation (2.18) between $P_{\nu_{2} \rightarrow \nu_{e}}^{\text {Earth }}$ and $P_{\nu_{2} \rightarrow \nu_{s}}^{\text {Earth }}$. In order to see the reason of this relation, it is useful to consider the most general $4 \times 4$ mixing matrix (without $C P$-violating phases) given in Eq. (2.3), that can be written as

$$
U=U^{\prime} U_{12}
$$

with

$$
U^{\prime}=U_{34} U_{24} U_{23} U_{14} U_{13} .
$$

Let us define the neutrino states

$$
\left|\nu_{r}^{\prime}\right\rangle=\sum_{\alpha=e, s, \mu, \tau} U_{\alpha r}^{\prime}\left|\nu_{\alpha}\right\rangle \quad(r=1,2,3,4)
$$

The amplitudes of $\nu_{k} \rightarrow \nu_{\alpha}$ transitions in the Earth for $k$ $=1,2$ are given by

$$
A_{\nu_{k} \rightarrow \nu_{\alpha}}^{\text {Earth }}=\left\langle\nu_{\alpha}|\mathcal{S}| \nu_{k}\right\rangle=\sum_{r=1}^{4}\left\langle\nu_{\alpha} \mid \nu_{r}^{\prime}\right\rangle\left\langle\nu_{r}^{\prime}|\mathcal{S}| \nu_{k}\right\rangle=\sum_{r=1}^{4} U_{\alpha r}^{\prime} S_{r k}^{\prime},
$$

where the unitary operator $\mathcal{S}$ describes the evolution inside the Earth and $S_{r k}^{\prime} \equiv\left\langle\nu_{r}^{\prime}|\mathcal{S}| \nu_{k}\right\rangle$.

It has been shown in Ref. [22] that the matter effects inside the Earth can generate only transitions between $\nu_{1}, \nu_{2}$ and $\nu_{1}^{\prime}, \nu_{2}^{\prime}$. Then, we have

$$
\begin{gathered}
A_{\nu_{k} \rightarrow v_{\alpha}}^{\text {Earth }}=U_{\alpha 1}^{\prime} S_{1 k}^{\prime}+U_{\alpha 2}^{\prime} S_{2 k}^{\prime} \quad(k=1,2), \\
A_{v_{k} \rightarrow v_{\alpha}}^{\text {Earth }}=U_{\alpha k}^{\prime}=U_{\alpha k} \quad(k=3,4),
\end{gathered}
$$

and the transition probabilities are given by

$$
\begin{aligned}
P_{\nu_{k} \rightarrow \nu_{\alpha}}^{\text {Earth }}= & U_{\alpha 1}^{\prime 2}\left|S_{1 k}^{\prime}\right|^{2}+U_{\alpha 2}^{\prime 2}\left|S_{2 k}^{\prime}\right|^{2} \\
& +2 U_{\alpha 1}^{\prime} U_{\alpha 2}^{\prime} \operatorname{Re}\left[S_{1 k}^{\prime} S_{2 k}^{\prime *}\right] \quad(k=1,2), \\
& P_{\nu_{k} \rightarrow \nu_{\alpha}}^{\text {Earth }}=U_{\alpha k}^{2} \quad(k=3,4) .
\end{aligned}
$$

Since the evolution operator $\mathcal{S}$ is unitary, the matrix $S^{\prime}$ is unitary and we have the relations

$$
\begin{gathered}
\left|S_{12}^{\prime}\right|^{2}=\left|S_{21}^{\prime}\right|^{2} \equiv P_{12}^{\prime}, \quad\left|S_{11}^{\prime}\right|^{2}=\left|S_{22}^{\prime}\right|^{2}=1-P_{12}^{\prime}, \\
S_{11}^{\prime} S_{21}^{\prime *}+S_{12}^{\prime} S_{22}^{\prime *}=0,
\end{gathered}
$$

where $P_{12}^{\prime}$ is the probability of $\nu_{2} \leftrightarrows \nu_{1}^{\prime}$ transitions, that is equal to the probability of $\nu_{1} \leftrightarrows \nu_{2}^{\prime}$ transitions. It is easy to 
check that the unitarity constraints (A9) are equivalent to the probability conservation relations

$$
\sum_{k=1}^{4} P_{\nu_{k} \rightarrow \nu_{\alpha}}^{\text {Earth }}=1, \sum_{\alpha=e, s, \mu, \tau} P_{\nu_{k} \rightarrow \nu_{\alpha}}^{\text {Earth }}=1 .
$$

Let us notice that by construction the matrix $U^{\prime}$ is such that

$$
U_{e 2}^{\prime}=0
$$

and the probability of $\nu_{k} \rightarrow \nu_{e}$ transitions inside the Earth depend only on $U_{e 1}^{\prime 2}=\cos \vartheta_{13}^{2} \cos \vartheta_{14}^{2}$ and $P_{12}^{\prime}$ :

$$
P_{\nu_{1} \rightarrow \nu_{e}}^{\text {Earth }}=U_{e 1}^{\prime 2}\left(1-P_{12}^{\prime}\right), \quad P_{\nu_{2} \rightarrow \nu_{e}}^{\text {Earth }}=U_{e 1}^{\prime 2} P_{12}^{\prime} \text {. }
$$

On the other hand, in general, for a given mixing matrix $U$, the transition probabilities $P_{\nu_{k} \rightarrow v_{\alpha}}^{\text {Earth }}$ with $\alpha=s, \mu, \tau$ depend on two independent quantities, $P_{12}^{\prime}$ and $\operatorname{Arg}\left[S_{11}^{\prime} S_{21}^{\prime *}\right]$. Hence, in general the probabilities $P_{\nu_{k} \rightarrow \nu_{e}}^{\text {Earth }}$ and $P_{\nu_{k} \rightarrow \nu_{s}}^{\text {Earth }}$ must be calculated independently. However, if

$$
U_{\alpha 1}^{\prime} U_{\alpha 2}^{\prime}=0 \quad(\alpha=s, \mu, \tau),
$$

also $P_{\nu_{k} \rightarrow \nu_{\alpha}}^{\text {Earth }}$ depends only on the elements of the mixing matrix and $P_{12}^{\prime}$ :

$$
\begin{aligned}
& P_{\nu_{1} \rightarrow \nu_{\alpha}}^{\text {Earth }}=U_{\alpha 1}^{\prime 2}\left(1-P_{12}^{\prime}\right)+U_{\alpha 2}^{\prime 2} P_{12}^{\prime}, \\
& P_{\nu_{2} \rightarrow \nu_{\alpha}}^{\text {Earth }}=U_{\alpha 1}^{\prime 2} P_{12}^{\prime}+U_{\alpha 2}^{\prime 2}\left(1-P_{12}^{\prime}\right),
\end{aligned}
$$

for $\alpha=s, \mu, \tau$.
In the approximation $\vartheta_{13}=\vartheta_{14}=0$ that we use in the analysis of solar neutrino data, we have

$$
U_{e 1}^{\prime}=1, \quad U_{\alpha 1}^{\prime}=0 \quad(\alpha=s, \mu, \tau)
$$

Therefore, condition (A13) is satisfied and we obtain

$$
\begin{gathered}
P_{\nu_{1} \rightarrow \nu_{e}}^{\text {Earth }}=1-P_{12}^{\prime}, \quad P_{\nu_{2} \rightarrow \nu_{e}}^{\text {Earth }}=P_{12}^{\prime}, \\
P_{\nu_{1} \rightarrow \nu_{\alpha}}^{\text {Earth }}=U_{\alpha 2}^{\prime 2} P_{12}^{\prime}, \quad P_{\nu_{2} \rightarrow \nu_{\alpha}}^{\text {Earth }}=U_{\alpha 2}^{\prime 2}\left(1-P_{12}^{\prime}\right) .
\end{gathered}
$$

Eliminating $P_{12}^{\prime}$ from relations (A16) and (A17), we obtain

$$
P_{\nu_{k} \rightarrow \nu_{\alpha}}^{\text {Earth }}=U_{\alpha 2}^{\prime 2}\left(1-P_{\nu_{k} \rightarrow \nu_{e}}^{\text {Earth }}\right) \quad(k=1,2 ; \quad \alpha=s, \mu, \tau) .
$$

In particular, for $k=2$ and $\alpha=s$, we obtain the useful relation (2.18) between $P_{\nu_{2} \rightarrow \nu_{s}}^{\text {Earth }}$ and $P_{\nu_{2} \rightarrow \nu_{e}}^{\text {Earth }}$.

In general, considering the possibility of small but nonzero $\vartheta_{13}$ and/or $\vartheta_{14}$, if the mixing angles are such that $U_{\alpha 1}^{\prime}=0$ for $\alpha=s, \mu, \tau$, we have the relation

$$
P_{\nu_{k} \rightarrow \nu_{\alpha}}^{\text {Earth }}=U_{\alpha 2}^{\prime 2}\left(1-\frac{P_{\nu_{k} \rightarrow \nu_{e}}^{\text {Earth }}}{U_{e 1}^{\prime 2}}\right) \quad(\alpha=s, \mu, \tau),
$$

whereas if $U_{\alpha 2}^{\prime}=0$ we obtain the relation

$$
P_{\nu_{k} \rightarrow \nu_{\alpha}}^{\text {Earth }}=\frac{U_{\alpha 1}^{\prime 2}}{U_{e 1}^{\prime 2}} P_{\nu_{k} \rightarrow \nu_{e}}^{\text {Earth }} \quad(\alpha=s, \mu, \tau) .
$$

[1] R. Davis, Jr., D. S. Harmer, and K. C. Hoffman, Phys. Rev. Lett. 20, 1205 (1968).

[2] J. N. Bahcall, N. A. Bahcall, and G. Shaviv, Phys. Rev. Lett. 20, 1209 (1968); J. N. Bahcall and R. Davis, Jr., Science 191, 264 (1976).

[3] J. N. Bahcall, M. H. Pinsonneault, S. Basu, and J. ChristensenDalsgaard, Phys. Rev. Lett. 78, 171 (1997).

[4] J. N. Bahcall and M. H. Pinsonneault, Rev. Mod. Phys. 67, 781 (1995).

[5] B. T. Cleveland et al., Astrophys. J. 496, 505 (1998).

[6] T. Kirsten, Talk at the Sixth international workshop on topics in astroparticle and underground physics September, TAUP99, Paris, 1999.

[7] SAGE Collaboration, J. N. Abdurashitov et al., Phys. Rev. C 60, 055801 (1999).

[8] Kamiokande Collaboration, Y. Fukuda et al., Phys. Rev. Lett. 77, 1683 (1996).

[9] Super-Kamiokande Collaboration, Y. Fukuda et al., Phys. Rev. Lett. 82, 1810 (1999); 82, 2430 (1999).

[10] Y. Suzuki, talk a the "XIX International Symposium on Lepton and Photon Interactions at High Energies,' Stanford University, 1999; M. Nakahata, talk at the "6th International
Workshop on Topics in Astroparticle and Underground Physics, TAUP99," Paris, 1999.

[11] P. C. de Holanda, C. Peña-Garay, M. C. Gonzalez-Garcia, and J. W. F. Valle, Phys. Rev. D 60, 093010 (1999).

[12] M. C. Gonzalez-Garcia and A. Yu. Smirnov, in Proceedings of International Workshop on Particles in Astrophysics and Cosmology: on Theory to Observation, Valencia, 1999, edited by V. Berezinsky, G. Raffelt, and J. W. F. Valle [Nucl. Phys. B (Proc. Suppl.) (in press)] (http://flamenco.uv.es//v99.html)

[13] V. N. Gribov and B. M. Pontecorvo, Phys. Lett. 28B, 493 (1969); V. Barger, K. Whisnant, and R. J. N. Phillips, Phys. Rev. D 24, 538 (1981); S. L. Glashow and L. M. Krauss, Phys. Lett. B 190, 199 (1987); V. Barger, R. J. Phillips, and K. Whisnant, Phys. Rev. Lett. 65, 3084 (1990); S. L. Glashow, P. J. Kernan, and L. M. Krauss, Phys. Lett. B 445, 412 (1999); V. Berezinsky, G. Fiorentini, and M. Lissia, hep-ph/9811352; hep-ph/9904225.

[14] S. P. Mikheyev and A. Yu. Smirnov, Yad. Fiz. 42, 1441 (1985) [Sov. J. Nucl. Phys. 42, 913 (1985)]; L. Wolfenstein, Phys. Rev. D 17, 2369 (1978).

[15] NUSEX Collaboration, M. Aglietta et al., Europhys. Lett. 8, 611 (1989); Fréjus Collaboration, Ch. Berger et al., Phys. Lett. 
B 227, 489 (1989); IMB Collaboration, D. Casper et al., Phys. Rev. Lett. 66, 2561 (1991); R. Becker-Szendy et al., Phys. Rev. D 46, 3720 (1992); Kamiokande Collaboration, H. S. Hirata et al., Phys. Lett. B 205, 416 (1988); 280, 146 (1992); Kamiokande Collaboration, Y. Fukuda et al., ibid. 335, 237 (1994); Soudan Collaboration, W. W. M. Allison et al., ibid. 391, 491 (1997).

[16] Super-Kamiokande Collaboration, Y. Fukuda et al., Phys. Rev. Lett. 81, 1562 (1998); 82, 2644 (1999).

[17] C. Athanassopoulos, Phys. Rev. Lett. 75, 2650 (1995); 77, 3082 (1996); 81, 1774 (1998).

[18] J. T. Peltoniemi, D. Tommasini, and J. W. F. Valle, Phys. Lett. B 298, 383 (1993); E. J. Chun et al., ibid. 357, 608 (1995); S. C. Gibbons et al., ibid. 430, 296 (1998); B. Brahmachari and R. N. Mohapatra, ibid. 437, 100 (1998); S. Mohanty, D. P. Roy, and U. Sarkar, ibid. 445, 185 (1998); J. T. Peltoniemi and J. W. F. Valle, Nucl. Phys. B406, 409 (1993); W. Y. Liu and A. Yu. Smirnov, ibid. B524, 505 (1998); D. O. Caldwell, Int. J. Mod. Phys. A 13, 4409 (1998); D. O. Caldwell and R. N. Mohapatra, Phys. Rev. D 48, 3259 (1993); E. Ma and P. Roy, ibid. 52, R4780 (1995); A. Yu. Smirnov and M. Tanimoto, ibid. 55, 1665 (1997); N. Gaur et al., ibid. 58, 071301 (1998); E. J. Chun, C. W. Kim, and U. W. Lee, ibid. 58, 093003 (1998); K. Banakli and A. Yu. Smirnov, Phys. Rev. Lett. 79, 4314 (1997); Y. Chikira, N. Haba, and Y. Mimura, hep-ph/9808254; C. Liu and J. Song, Phys. Rev. D 60, 036002 (1999); W. Grimus, R. Pfeiffer, and T. Schwetz, hep-ph/9905320.

[19] J. J. Gomez-Cadenas and M. C. Gonzalez-Garcia, Z. Phys. C 71, 443 (1996); N. Okada and O. Yasuda, Int. J. Mod. Phys. A 12, 3669 (1997); S. Goswami, Phys. Rev. D 55, 2931 (1997); S. M. Bilenky, C. Giunti, and W. Grimus, ibid. 57, 1920 (1998); 58, 033001 (1998); S. M. Bilenky, C. Giunti, W. Grimus, and T. Schwetz, Astropart. Phys. 11, 413 (1999); V. Barger, Y. B. Dai, K. Whisnant, and B. L. Young, Phys. Rev. D 59, 113010 (1999); V. Barger, T. J. Weiler, and K. Whisnant, Phys. Lett. B 427, 97 (1998); C. Giunti, Phys. Rev. D 61, 036002 (2000); C. Giunti, Phys. Lett. B 467, 83 (1999); S. M. Bilenky et al., ibid. 465, 193 (1999); C. Giunti, J. High Energy Phys. 01, 032 (2000); A. Ibarra and I. Navarro, hep-ph/9912282.

[20] S. M. Bilenky, C. Giunti, and W. Grimus, Eur. Phys. J. C 1, 247 (1998); Proceedings of Neutrino '96, Helsinki, June 1996, edited by K. Enqvist et al., (World Scientific, Singapore, 1997), p. 174; hep-ph/9609343; S. M. Bilenky, C. Giunti, W. Grimus, and T. Schwetz, Phys. Rev. D 60, 073007 (1999).

[21] V. Barger, S. Pakvasa, T. J. Weiler, and K. Whisnant, Phys. Rev. D 58, 093016 (1998).
[22] D. Dooling, C. Giunti, K. Kang, and C. W. Kim, Phys. Rev. D 61, 073011 (2000).

[23] J. Bouchez et al., Z. Phys. C 32, 499 (1986); S. P. Mikheyev and A. Yu. Smirnov, in '86 Massive Neutrinos in Astrophysics and in Particle Physics, proceedings of the Sixth Moriond Workshop, edited by O. Fackler and J. Trân Thanh Vân (Editions Frontières, Gif-sur-Yvette, 1986), p. 355; S. P. Mikheyev and A. Yu. Smirnov, Usp. Fiz. Nauk 153, 3 (1987) [Sov. Phys. Usp. 30, 759 (1987)]; A. Dar et al., Phys. Rev. D 35, 3607 (1987); E. D. Carlson, ibid. 34, 1454 (1986); A. J. Baltz and J. Weneser, ibid. 50, 5971 (1994); A. J. Baltz and J. Weneser, ibid. 51, 3960 (1994); P. I. Krastev, hep-ph/9610339; Q. Y. Liu, M. Maris, and S. T. Petcov, Phys. Rev. D 56, 5991 (1997); 56, 7444 (1997); J. N. Bahcall and P. I. Krastev, Phys. Rev. C 56, 2839 (1997); A. J. Baltz and J. Weneser, Phys. Rev. D 35, 528 (1987); 37, 3364 (1988); E. Lisi and D. Montanino, ibid. 56, 1792 (1997); S. T. Petcov, Phys. Lett. B 434, 321 (1998); M. Chizhov, M. Maris, and S. T. Petcov, hep-ph/9810501; M. V. Chizhov and S. T. Petcov, Phys. Rev. Lett. 83, 1096 (1999); A. S. Dighe, Q. Y. Liu, and A. Yu. Smirnov, hep-ph/9903329; A. H. Guth, L. Randall, and M. Serna, J. High Energy Phys. 8, 018 (1999).

[24] E. K. Akhmedov, Nucl. Phys. B538, 25 (1999).

[25] S. M. Bilenky, C. Giunti, and W. Grimus, Prog. Part. Nucl. Phys. 43, 1 (1999).

[26] G. L. Fogli, E. Lisi, D. Montanino, and A. Palazzo, hep-ph/9912231.

[27] J. N. Bahcall, S. Basu, and M. Pinsonneault, Phys. Lett. B 433, 1 (1998).

[28] http://www.sns.ias.edu/ jnb/SNdata

[29] B. Faid, G. L. Fogli, E. Lisi, and D. Montanino, Phys. Rev. D 55, 1353 (1997).

[30] J. N. Bahcall, M. Kamionkowsky, and A. Sirlin, Phys. Rev. D 51, 6146 (1995).

[31] G. L. Fogli, E. Lisi, and D. Montanino, Phys. Rev. D 49, 3626 (1994); G. L. Fogli and E. Lisi, Astropart. Phys. 3, 185 (1995).

[32] M. C. Gonzalez-Garcia, P. C. de Holanda, C. Peña-Garay, and J. W. F. Valle, hep-ph/9906469.

[33] B. Achkar et al., Nucl. Phys. B424, 503 (1995).

[34] CHOOZ Collaboration, M. Apollonio et al., Phys. Lett. B 420, 397 (1998).

[35] Andre de Gouvea, Alexander Friedland, and Hitoshi Murayama, hep-ph/9910286.

[36] M. C. Gonzalez-Garcia, H. Nunokawa, O. L. G. Peres, T. Stanev, and J. W. F. Valle, Phys. Rev. D 58, 033004 (1998); M. C. Gonzalez-Garcia, H. Nunokawa, O. L. Peres, and J. W. F. Valle, Nucl. Phys. B543, 3 (1999); for a recent experimental review, see W. Anthony Mann, Plenary talk at the XIX Int. Symposium on Lepton and Photon Interactions at High Energies, Stanford, 1999, hep-ex/9912007. 